\title{
Fin56-induced ferroptosis is supported by autophagy-mediated GPX4 degradation and functions synergistically with mTOR inhibition to kill bladder cancer cells
}

\author{
Yadong Sun ${ }^{1}$, Niklas Berleth ${ }^{1}$, Wenxian Wu ${ }^{1}$, David Schlütermann ${ }^{1}$, Jana Deitersen ${ }^{1}$, Fabian Stuhldreier ${ }^{1}$, Lena Berning ${ }^{1}$, \\ Annabelle Friedrich ${ }^{1}$, Seda Akgün ${ }^{1}$, María José Mendiburo (iD) ${ }^{1}$, Sebastian Wesselborg ${ }^{1}$, Marcus Conrad (iD ${ }^{2,3}$, Carsten Berndt (iD) ${ }^{4}$ and \\ Björn Stork (iD) ${ }^{1 凶}$
}

(c) The Author(s) 2021

Ferroptosis is a form of regulated cell death that emerges to be relevant for therapy-resistant and dedifferentiating cancers. Although several lines of evidence suggest that ferroptosis is a type of autophagy-dependent cell death, the underlying molecular mechanisms remain unclear. Fin56, a type 3 ferroptosis inducer, triggers ferroptosis by promoting glutathione peroxidase 4 (GPX4) protein degradation via a not fully understood pathway. Here, we determined that Fin56 induces ferroptosis and autophagy in bladder cancer cells and that Fin56-triggered ferroptosis mechanistically depends on the autophagic machinery. Furthermore, we found that autophagy inhibition at different stages attenuates Fin56-induced oxidative stress and GPX4 degradation. Moreover, we investigated the effects of Fin56 in combination with Torin 2, a potent mTOR inhibitor used to activate autophagy, on cell viability. We found that Fin56 synergizes with Torin 2 in cytotoxicity against bladder cancer cells. Collectively, our findings not only support the concept that ferroptosis is a type of autophagy-dependent cell death but imply that the combined application of ferroptosis inducers and mTOR inhibitors is a promising approach to improve therapeutic options in the treatment of bladder cancer.

Cell Death and Disease (2021)12:1028; https://doi.org/10.1038/s41419-021-04306-2

\section{INTRODUCTION}

Bladder cancer (BC) is the 10th most-common cancer worldwide, with an estimated 549,000 new cases and 200,000 deaths each year [1]. Recently, tumor genomic profiling supports the identification of promising therapeutic targets [2, 3]. Among them, the phosphatidylinositol 3-kinase (PI3K)/AKT/mammalian target of rapamycin (mTOR) pathway regulates various processes, including proliferation, survival, and autophagy [4, 5]. In up to $40 \%$ of $\mathrm{BCs}, \mathrm{mTOR}$ signaling is activated and related to tumor progression $[6,7]$. Whereas many mTOR inhibitors are in different phases of (pre-)clinical trials [8], the monotherapy of these inhibitors has not yet led to effective, desirable outcomes.

Ferroptosis, an iron-dependent form of regulated cell death, is entirely distinct from other cell death modalities [9]. Although ferroptosis has been linked to various pathological scenarios, it has received extensive attention in cancer therapy research [10]. Since cancer cells require higher levels of iron, generate higher levels of reactive oxygen species (ROS), and have an altered lipid metabolism compared with normal cells, they become more susceptible to ferroptosis [11]. So far, three types of inducers are available, which employ different modes of action [12]. These compounds have in common that they inhibit glutathione peroxidase 4 (GPX4), which itself represents a key inhibitor of phospholipid peroxidation [13]. GPX4 inhibition can be achieved either indirectly via depletion of glutathione by inhibiting cysteine uptake (e.g., type 1 inducer erastin) or directly (e.g., type 2 inducer RAS-selective lethal 3, RSL3). Fin56, a recently developed type 3 inducer, triggers ferroptosis by promoting degradation of GPX4 via a not fully understood pathway [14]. Accumulating evidence suggests that ferroptosis is related to another important cellular process, i.e., autophagy $[15,16]$.

Autophagy has an important role in transporting unfolded proteins and damaged organelles into lysosomes to be recycled [17]. Autophagy can be subdivided into three morphologically distinct types: macroautophagy, microautophagy, and chaperonemediated autophagy (CMA) [18]. The machinery regulating macroautophagy (hereafter, referred to as autophagy) includes the unc-51-like kinase 1 (ULK1) complex, the class III PI3K lipid kinase complex, and two ubiquitin-like conjugation systems [19]. mTOR is one of the main upstream regulators of autophagy. It phosphorylates ULK1 at Ser758 (Ser757 in murine ULK1) and thereby suppresses autophagy [20]. Once mTOR is pharmacologically inhibited or cells are kept under starvation conditions, this ULK1 site becomes rapidly dephosphorylated, leading to ULK1 activation and the induction of autophagy [21]. Although autophagy is generally considered a pro-survival mechanism, its

\footnotetext{
${ }^{1}$ Institute of Molecular Medicine I, Medical Faculty, Heinrich Heine University, Düsseldorf, Germany. ${ }^{2}$ Institute of Metabolism and Cell Death, Helmholtz Zentrum München, Neuherberg, Germany. ${ }^{3}$ Pirogov Russian National Research Medical University, Laboratory of Experimental Oncology, Ostrovityanova 1, Moscow 117997, Russia. ${ }^{4}$ Department of Neurology, Medical Faculty, Heinrich Heine University, Düsseldorf, Germany. ${ }^{凶}$ email: bjoern.stork@hhu.de Edited by: Professor Gian Maria Fimia
} 
role in cancer biology is more complex [22, 23]. In brief, autophagy is considered to execute a protective function during tumorigenesis; once tumor cells are established, autophagy supports tumor survival and metastasis. Moreover, with the development of methods to monitor autophagic flux in various contexts, autophagy-dependent cell death is considered as a genuine death pathway, making the relationship between autophagy and cancer biology even more complex [24, 25].

Recent evidence suggests that autophagy facilitates ferroptosis via the selective removal of ferroptosis-regulating molecules [26-28]. These pathways include NCOA4-mediated ferritinophagy, RAB7A-mediated lipophagy, or p62/SQSTM1-mediated clockophagy $[26,28]$. Furthermore, it has been observed that erastin and RSL3 induce autophagic flux and also affect GPX4 levels $[26,28]$. It has been reported that erastin promotes the degradation of GPX4 via CMA [29], and that RSL3 can block mTOR activation and cause GPX4 degradation in pancreatic cancer cells [30]. Along these lines, pharmacologic inhibition of mTORC1 decreases GPX4 protein levels [31], further supporting the relationship between autophagy and ferroptosis.

It has been described that Fin56 promotes the reduction of GPX4 but the mechanism or a possible link to autophagy remain unclear [14, 32]. Here, we found that Fin56 initiates ferroptosis and autophagy in BC cells. We observed that Fin56-induced autophagy promotes the degradation of GPX4 and ferritin, whereas the inhibition of autophagy dampened Fin56-induced lipid peroxidation and ferroptosis. In addition, we provide evidence that the combination of Fin56 with the mTOR inhibitor Torin 2 has a synergistic effect in effectively killing BC cells. In sum, we propose that the combined use of ferroptosis inducers and mTOR inhibitors is a promising approach to improve therapeutic options in the treatment of $B C$.

\section{RESULTS}

Fin56 induces autophagy-associated cell death in BC cells

It has been reported that the susceptibility to ferroptosis varies in different cancer cells [33, 34]. To evaluate the effects of ferroptosis inducers on human $B C$ cells, we employed type 1, 2, and 3 inducers (erastin, RSL3 and Fin56) and four BC cell lines representing different clinical stages and pathological grades of BC, i.e., J82, 253J, T24 and RT-112 cells (Figure S1A). All cell lines express GPX4 and solute carrier family 7 member 11 (SLC7A11) (Figure S1B). The cystine/glutamate antiporter system $\mathrm{x}_{\mathrm{c}}{ }^{-}$plays a key role in importing cystine for glutathione synthesis and thus protects from oxidative stress and ferroptosis [35]. The expression level of its subunit SLC7A11 directly correlates with the activity of the antiporter [36]. Owing to its mode of action, erastin failed to induce cell death in all tested cell lines after 3-9 h of treatment, whereas RSL3 and Fin56 triggered cell death in BC cells except for J82 (Fig. 1). After $24 \mathrm{~h}$, erastin induced cell death in 253J and T24 cells, and Fin56 induced cell death in all tested cell lines. Moreover, cell death caused by Fin56 was dampened by the autophagy inhibitor bafilomycin $A_{1}$ in 253J and T24 cells, indicating that Fin56 might trigger autophagy-associated cell death in these BC cells (Fig. 1B, C).

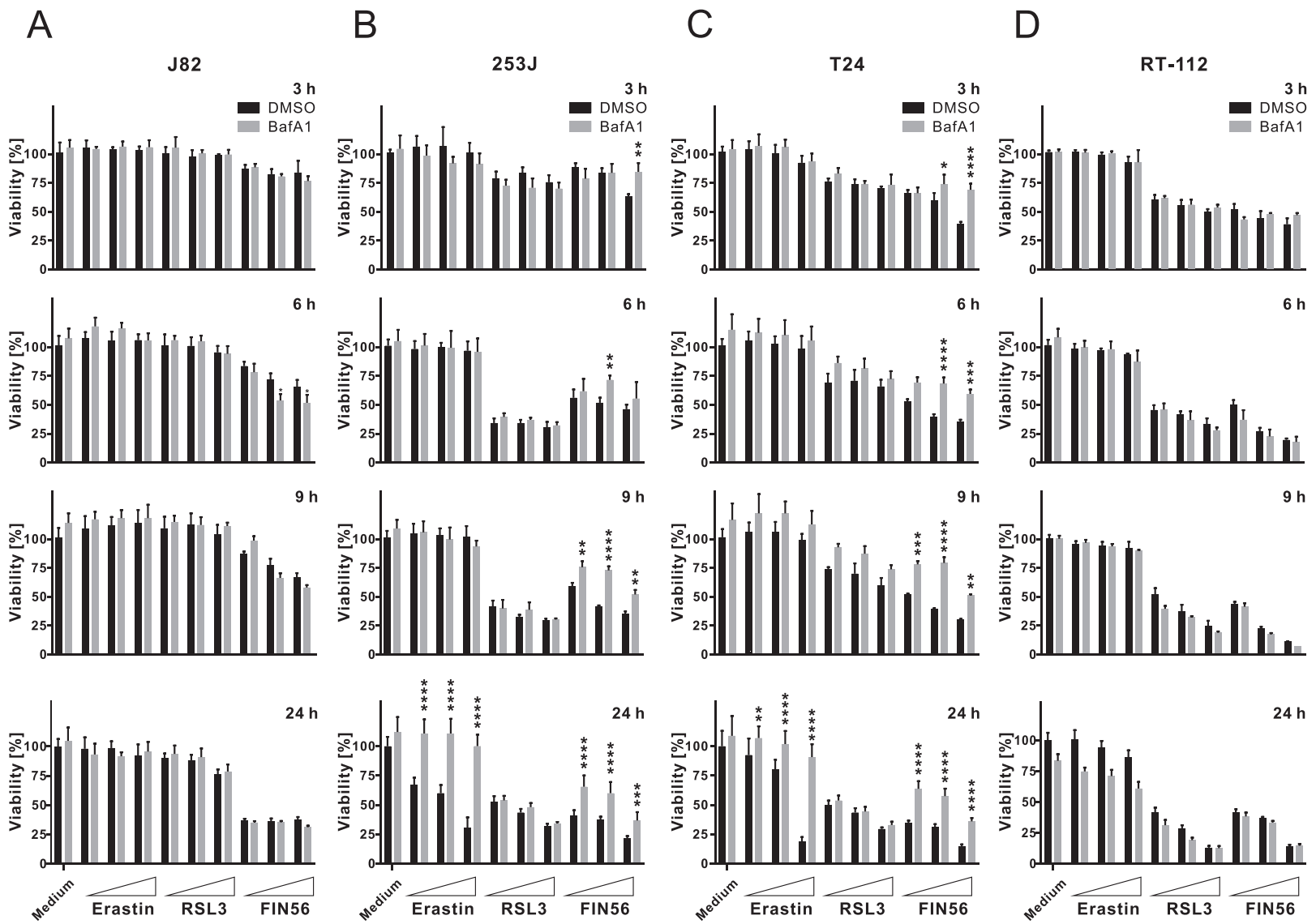

Fig. 1 Fin56 induces autophagy-associated cell death in bladder cancer cells. A-D J82, 253J, T24, and RT-112 bladder cancer cells were treated with different concentrations $(1 \mu \mathrm{M}, 2 \mu \mathrm{M}$, and $5 \mu \mathrm{M})$ of Erastin, RSL3, and Fin56 with or without $20 \mathrm{nM}$ bafilomycin $\mathrm{A}_{1}$ (BafA1) for different times $(3,6,9$, and $24 \mathrm{~h}$ ). After treatment, cell viability was measured using MTT assay (A-D represent the cell viability of J82, 253J, T24, and RT-112, respectively). Results are shown as means + SD of two independent experiments. $P$ values were determined by two-way ANOVA with Sidak's post hoc test. ${ }^{*} p<0.05 ;{ }^{* *} p<0.01 ;{ }^{* * *} p<0.001 ;{ }^{* * *} p<0.0001$ (comparison between DMSO- and BafA1-treated samples). 


\section{Fin56 induces ferroptosis in BC cells}

To further characterize Fin56-induced cell death, we treated BC cell lines with increasing concentrations of Fin56 for $72 \mathrm{~h}$. Fin56 conferred high cytotoxicity in all tested cell lines (Fig. 2A). In order to further investigate the mode of cell death, we next treated the four BC cell lines with Fin56 in the presence or absence of two different ferroptosis inhibitors (a-tocopherol and liproxstatin-1). Both inhibitors significantly prevented Fin56-induced cell death in all BC cell lines (Fig. 2B and S2).

Since Fin56 induces autophagy-associated cell death in 253J and T24 cells, we focused on these two cell lines. We established SLC7A11-overexpressing 253J and T24 cells and exposed them to different ferroptosis inducers. Compared to control cells, SLC7A11 overexpression inhibited cell death induced by the three inducers (Fig. 2C). Taken together, these results suggest that Fin56 induces ferroptosis in these BC cells.

\section{Fin56 induces autophagy in BC cells}

Since bafilomycin $A_{1}$ partially protected cells from Fin56-induced ferroptosis, we investigated whether Fin56 treatment induced autophagy. Autophagy was evaluated by determining the changes of the autophagosome marker LC3 and the autophagy receptor sequestosome 1 (SQSTM1/p62) by immunoblotting [37]. Levels of LC3-II, a membrane-associated form of LC3, were increased after Fin56 treatment in a time- and dose-dependent manner. Simultaneously, SQSTM1/p62 was decreased in the same manner (Fig. 3A, B). These results indicate that Fin56 treatment can induce autophagy in BC. However, the accumulation of LC3-II may represent either an increase in autophagosome generation and/or a block of autophagic flux. To further analyze this, we performed LC3 and SQSTM1/p62 turnover assays using bafilomycin $A_{1}$ to block lysosomal degradation of autophagosomes. We found that LC3-II levels increased under Fin56 treatment or bafilomycin $A_{1}$ treatment alone. However, the combined treatment with Fin56 and bafilomycin $A_{1}$ led to an increased accumulation of LC3-II (Fig. 3C). Similar results were obtained from the SQSTM1/p62 turnover assay. These results suggest that Fin56 increases autophagic flux. However, since LC3 and SQSTM1/ p62 can be transcriptionally regulated, autophagic flux should not only be assessed by their protein abundance [38]. Thus, we generated 235J and T24 cells stably expressing mRFP-EGFP-rLC3, and monitored autophagic flux by the detection of yellow dots (mRFP/EGFP colocalization, representing autophagosomes) and red dots (mRFP only, representing autolysosomes) using fluorescence microscopy [39]. Consistently, Fin56 treatment increased the number of both autophagosomes and autolysosomes, indicating increased autophagic flux in 253J and T24 cells (Fig. 3D).

Since mTOR is a central upstream regulator of autophagy [40], we next investigated whether mTOR was involved in Fin56induced autophagy. We analyzed mTOR phosphorylation at Ser2448 and ULK1 phosphorylation at Ser758, which both have been linked to autophagy inhibition [41, 42]. Fin56 treatment, however, did not affect phosphorylation levels of mTOR and ULK1 at these sites (Fig. 3E), indicating that Fin56 induces autophagy in $\mathrm{BC}$ through an mTOR-independent pathway.

\section{Fin56 induces autophagy-dependent ferroptosis}

To investigate the relationship between Fin56-induced autophagy and ferroptosis more comprehensively, we inhibited autophagy by using knockdown or/and knockout of ULK1 and ATG3 and investigated Fin56-induced ferroptosis. ULK1 depletion blocks autophagy initiation, while in the absence of ATG3 autophagy is impaired during autophagosome biogenesis. We found that siRNA-mediated knockdown of ULK1 decreased Fin56-induced ferroptosis compared with control siRNA (Fig. 4A). We also employed ulk1/ulk2 double-knockout (DKO) murine embryonic fibroblasts (MEFs) and ulk1/ulk2 DKO MEFs reconstituted with
MYC-ULK1 to confirm the siRNA results. Compared with reconstituted cells, ulk1/ulk2 DKO cells had higher cell viability after treatment with different concentrations of Fin56 (Fig. 4B). Moreover, autophagy deficiency has been confirmed in cells lacking ATG3, an E2-like enzyme mediating LC3 lipidation [43]. In line with the ULK1 results, atg3 KO cells were more viable than Atg3 WT cells upon Fin56 treatment (Fig. 4C). Taken together, these results suggest that Fin56 triggers autophagy-dependent ferroptosis.

\section{Autophagy inhibition attenuates Fin56-induced oxidative stress and lipid peroxidation}

Lipid peroxidation is one of the hallmarks of ferroptosis. Frequently, the 2',7'-dichlorofluorescein (DCF) assay is used to evaluate cellular oxidative stress. As noted by Karlsson et al. [44], the oxidation of $\mathrm{H}_{2}$ DCF to DCF requires the presence of both $\mathrm{H}_{2} \mathrm{O}_{2}$ and redox-active transition metals such as iron, and rather indicates the impact of hydroxyl radicals generated during Fenton-type reactions. Since non-enzymatic lipid peroxidation is driven by the Fenton reaction, we performed the DCF assay and observed that Fin56 treatment increased DCF-induced fluorescence (Fig. 5A). Moreover, autophagy inhibition at different stages by using either bafilomycin $A_{1}$ or the class III PI3K inhibitor SAR405 reduced Fin56-induced DCF fluorescence. We also monitored lipid peroxidation by flow cytometric analysis of BODIPY 581/591 C11 fluorescence. In both 253J and T24 cells, SAR405 and bafilomycin $A_{1}$ reversed the Fin56-induced increase in the $530 / 30 \mathrm{~nm}$ fluorescence (Fig. 5B), indicating that autophagy inhibition attenuates Fin56-induced lipid peroxidation. We also analyzed BODIPY 581/591 C11-stained cells by immunofluorescence. Notably, we observed "onion-like" structures in Fin56-treated cells that were not present in control-treated cells (Figure S3). In order to investigate the potential contribution of intracellular iron, we next analyzed the expression of ferritin, an intracellular iron storage protein that sequesters iron in a soluble/non-toxic form [45]. We found that Fin56 promoted ferritin degradation and that autophagy inhibition interfered with its degradation (Fig. 5C). Excess oxidative stress causes mitochondrial damage and triggers the selective clearance of damaged mitochondria by mitophagy [46]. However, Fin56 did not induce significant mitochondrial fragmentation or mitophagy, as observed by staining of TOM20 and LC3 by immunofluorescence (Fig. 5D). Collectively, Fin56 induces oxidative damage/lipid peroxidation that can be prevented by the inhibition of autophagy and does not affect mitochondrial integrity.

\section{Autophagy inhibition attenuates Fin56-induced GPX4 degradation}

It has been reported that one pathway of Fin56-triggered ferroptosis is executed via GPX4 degradation [14], but the mechanistic details remained to be understood. We employed FLAG-Strep-HA-GPX4 (FSH-GPX4)-expressing MEFs to further investigate the Fin56-mediated GPX4 regulation [47, 48]. In addition, we used the antioxidant a-tocopherol to exclude the possibility that changes in GPX4 abundance are downstream consequences of the increased formation of the hydroxyl radical. We observed that Fin56 decreased the abundance of both ferritin and GPX4 in a time-dependent manner which was insensitive to a-TOC (Fig. 6A). Interestingly, we also found that ferritin levels increased after $9 \mathrm{~h}$ of Fin56 treatment. We speculate that the late increase in ferritin abundance may represent a self-protective mechanism that promotes the conversion of cellular iron into a non-toxic form to alleviate prolonged oxidative damage. Next, we investigated if autophagy is involved in this Fin56-induced GPX4 degradation, and indeed, we found that autophagy inhibition by bafilomycin $A_{1}$ or SAR405 attenuated GPX4 degradation induced by Fin56 in FSH-GPX4 MEFs (Fig. 6B). As it has been reported that erastin can induce GPX4 degradation via CMA [29], we next 
A

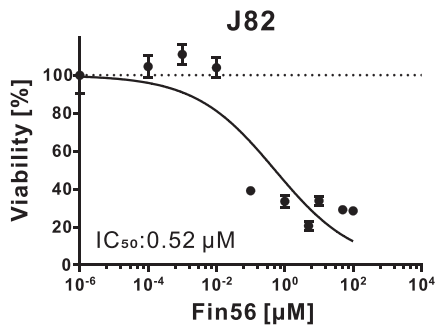

T24

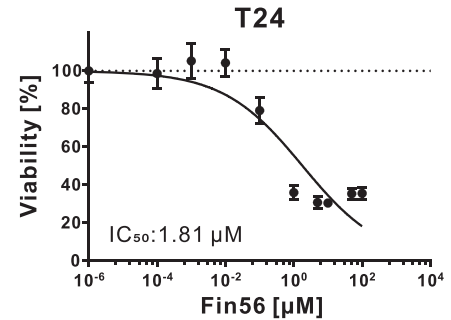

253J

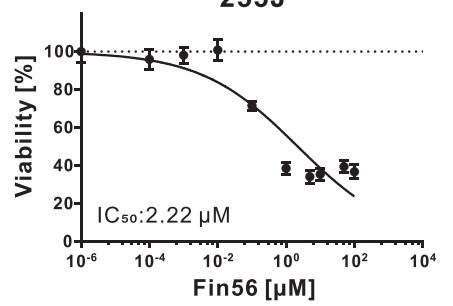

RT-112

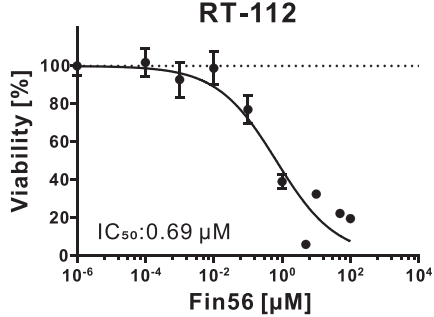

B

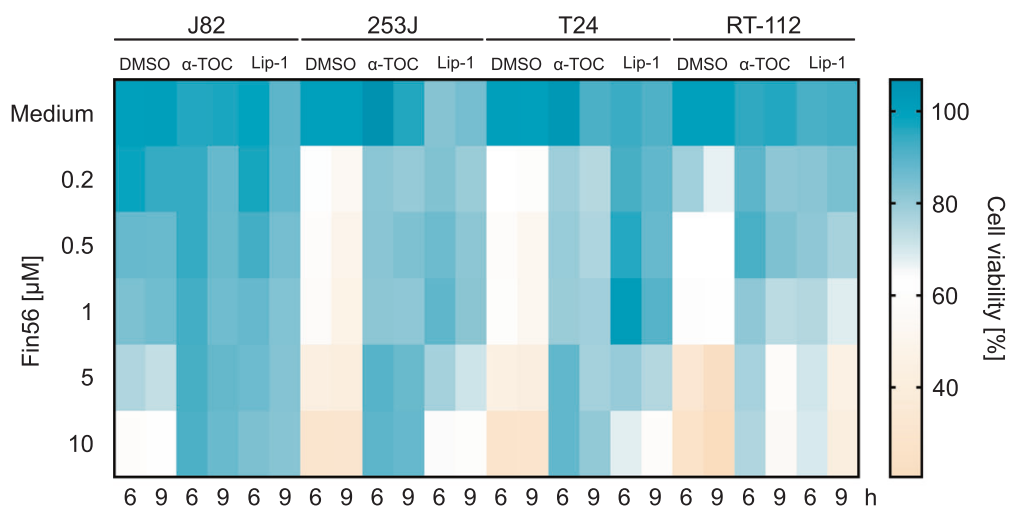

C

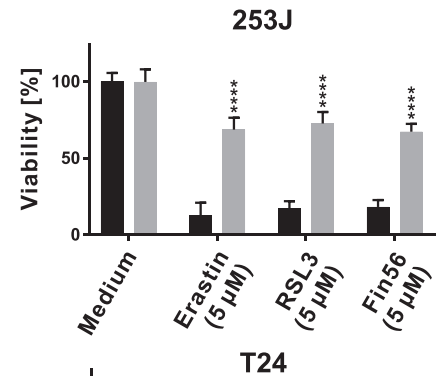

vector

HA-hSLC7A11
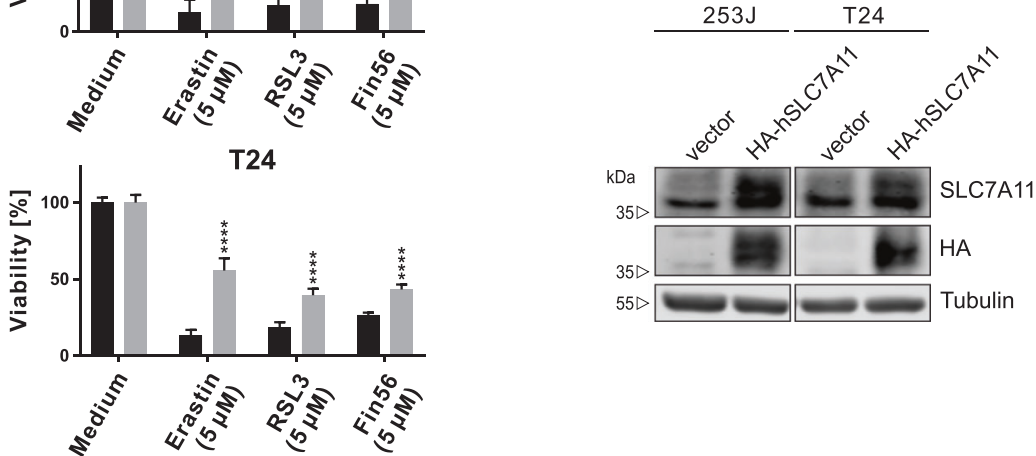

Fig. 2 Fin56 induces ferroptosis in bladder cancer cells. A J82, 253J, T24, and RT-112 bladder cancer cells were treated with different concentrations $(0.1 \mathrm{nM}-100 \mu \mathrm{M})$ of Fin56 for $72 \mathrm{~h}$. After treatment, cell viability was measured using MTT assay. Results are shown as means \pm SD of two independent experiments performed in quadruplicate for each treatment. B Cell viability heatmap of the effects of different ferroptosis inhibitors on cell death induced by Fin56. J82, 253J, T24, and RT-112 were treated with indicated concentrations of Fin56 with or without $\alpha$-tocopherol $(\alpha-$ TOC, $100 \mu \mathrm{M})$ or liproxstatin-1 (Lip-1, $500 \mathrm{nM})$ for 6 or $9 \mathrm{~h}$. After treatment, cell viability was measured using MTT assay. Results are from two independent experiments performed in triplicates for each treatment. The corresponding histograms and statistical analyses are shown in supplementary figure S1. C HA-hSLC7A11-overexpressing 253J or T24 cells and their corresponding vector control cells were treated with $5 \mu \mathrm{M}$ erastin, RSL3, or Fin56 for $24 \mathrm{~h}$. After treatment, cell viability was measured using MTT assay. Results are shown as means + SD of two independent experiments performed in triplicates for each treatment. $P$ values were determined by two-way ANOVA with Sidak's post hoc test. ${ }^{* * *} p<0.0001$ (comparison between vector- and HA-hSLC7A11-transfected cells). In order to confirm the overexpression of HA-hSLC7A11, cells were lysed and cellular lysates were immunoblotted for SLC7A11, HA, and tubulin. 
A

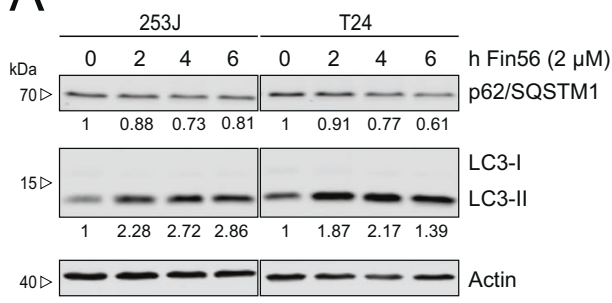

C

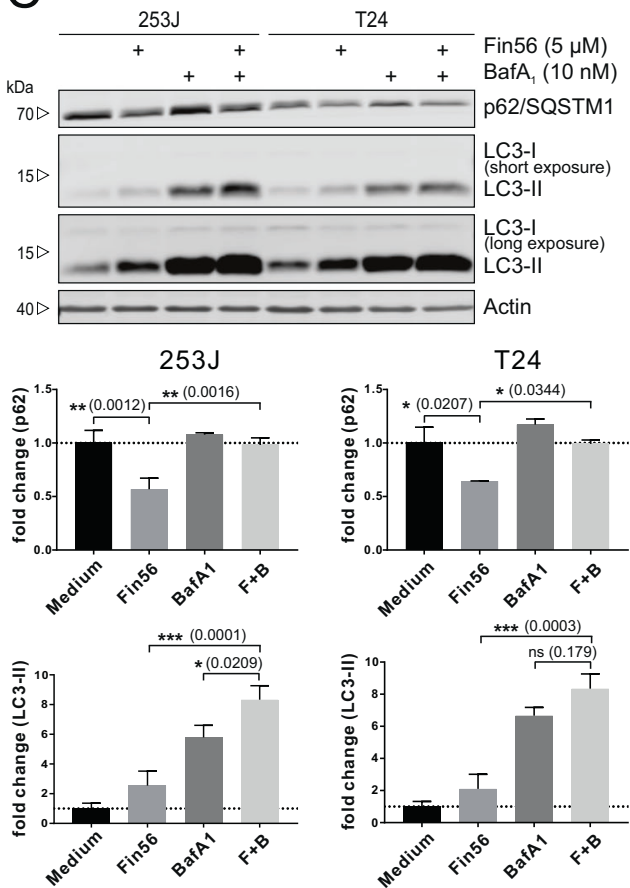

$\mathrm{E}$

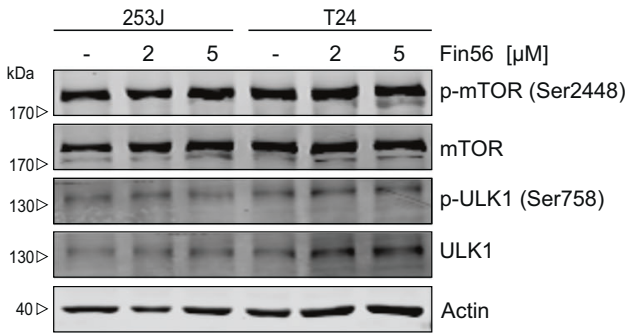

B

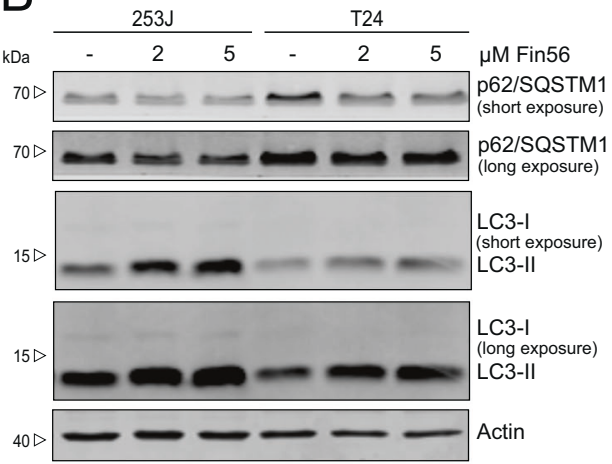

$\mathrm{D}$
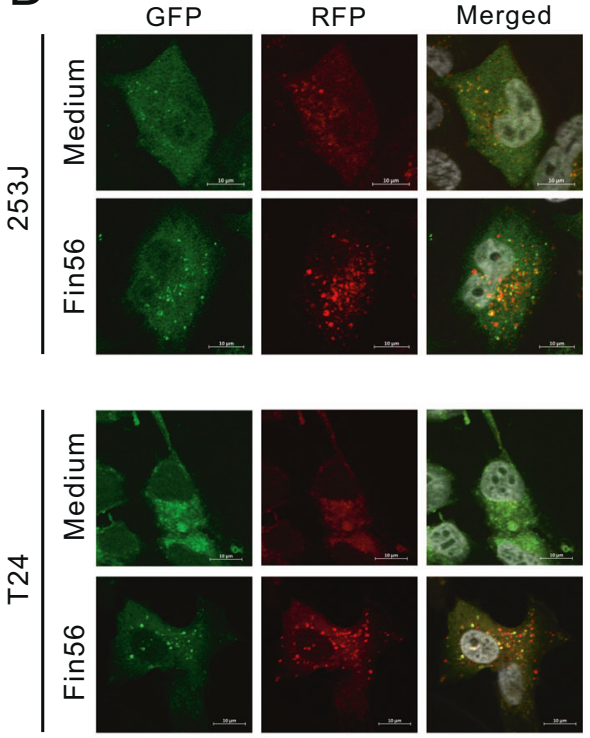

Autolysosome Autophagosome

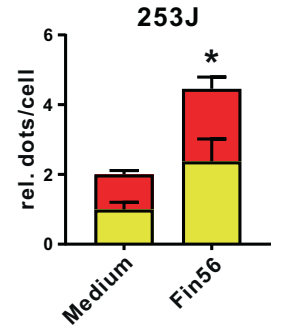

Fig. 3 Fin56 induces autophagy in bladder cancer cells. A 253J and T24 cells were treated with $2 \mu \mathrm{M}$ Fin56 for $0,2,4$, or $6 \mathrm{~h}$. After incubation, cells were lysed and cellular lysates were immunoblotted for the indicated proteins. One representative immunoblot is shown. The quantifications are from two independent experiments. B 253J and T24 cells were treated with 2 or $5 \mu \mathrm{M}$ Fin56 for $6 \mathrm{~h}$. After incubation, cells were lysed and cellular lysates were immunoblotted for the indicated proteins. One representative immunoblot of three independent experiments is shown. C 253J and T24 cells were treated with Fin56 $(5 \mu \mathrm{M})$, BafA1 (10 nM), or Fin56 + BafA1 (F+B) for $6 \mathrm{~h}$. After the incubation, cells were lysed and cellular lysates were immunoblotted for the indicated proteins. One representative immunoblot is shown. The quantifications of indicated ratios are from three independent experiments (means $+\mathrm{SD}$ ). The respective $P$ values are depicted in the diagram. D 253J and T24 stably expressing mRFP-EGFP-rLC3 cells were grown on glass coverslips one day prior to treatment. The following day, cells were treated with $2 \mu \mathrm{M}$ Fin 56 for $4 \mathrm{~h}$ and fixed after treatment. Red puncta represent autolysosomes and yellow puncta represent autophagosomes. One representative image is shown. Scale bars are $10 \mu \mathrm{m}$. The quantifications of puncta are from two independent experiments (means + SD); a minimum of 112 cells per stimulation was analyzed. The respective $P$ values are depicted in the diagram (comparison of $\mathrm{mRFP}^{+} \mathrm{EGFP}^{+}$puncta between medium- and Fin56-treated cells). E 253J and T24 cells were treated with $2 \mu \mathrm{M}$ or $5 \mu \mathrm{M}$ Fin56 for $6 \mathrm{~h}$. After the incubation, cells were lysed and cellular lysates were immunoblotted for the indicated proteins. One representative immunoblot of three independent experiments is shown. 


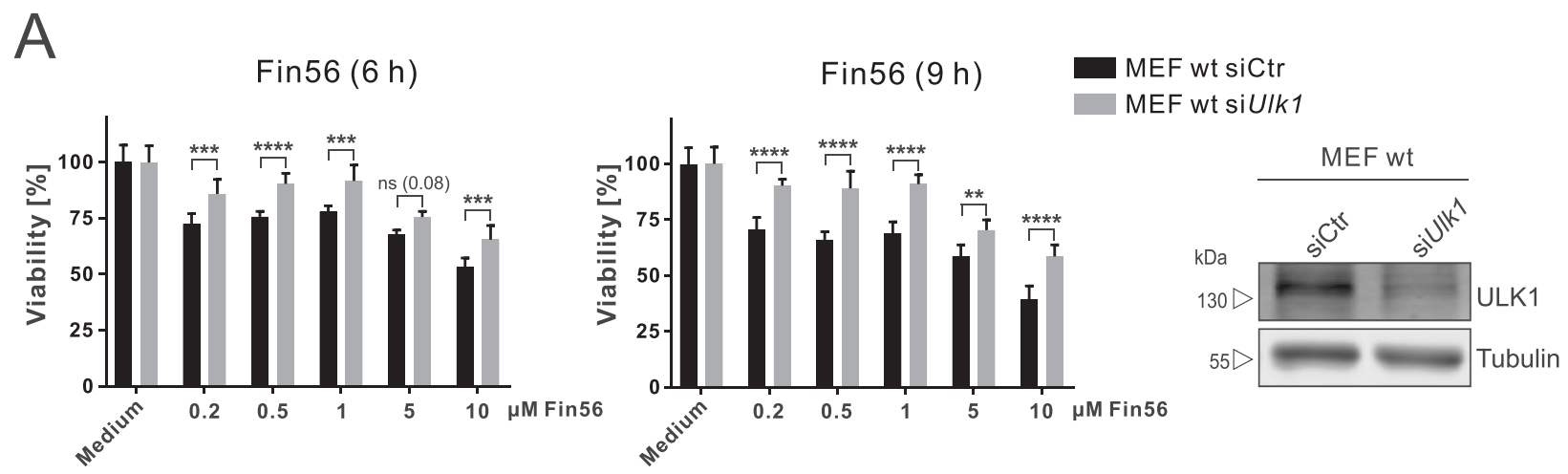

B
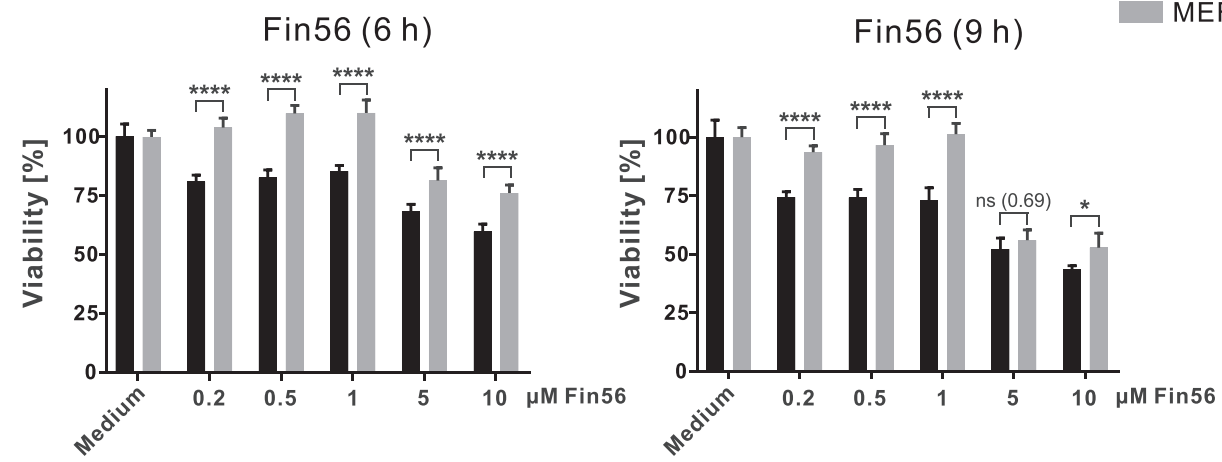

MEF ulk1/ulk2 DKO MYC-Ulk1

MEF ulk1/ulk2 DKO vector
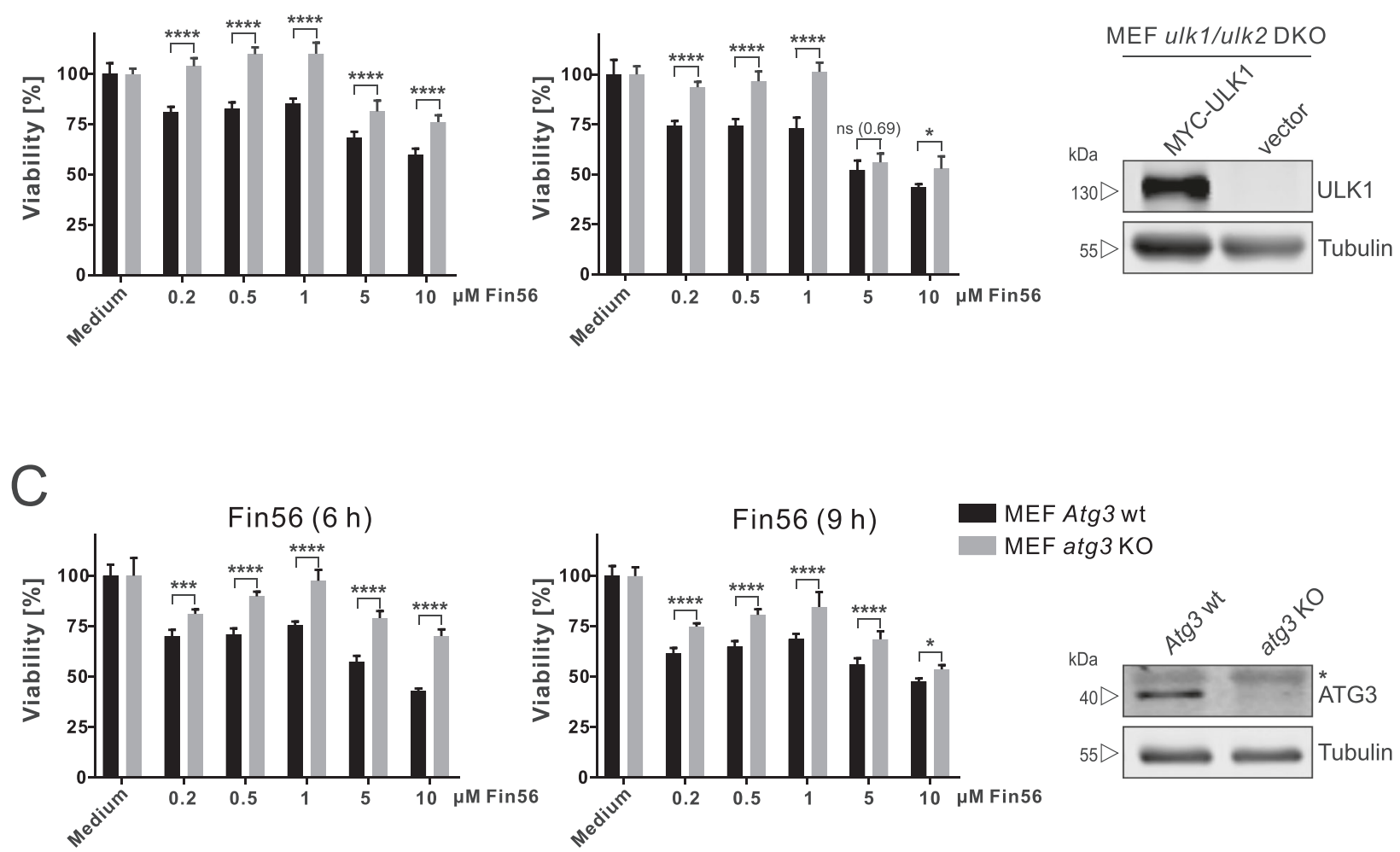

Fig. 4 Autophagy inhibition attenuates Fin56-induced ferroptosis. A MEFs were transfected with control or Ulk1 siRNAs. After $48 \mathrm{~h}$, cells were treated with different concentrations of Fin56 $(0.2 \mu \mathrm{M}-10 \mu \mathrm{M})$ for 6 or $9 \mathrm{~h}$. After treatment, cell viability was measured using MTT assay. Results are shown as means + SD of two independent experiments performed in triplicates for each treatment. $P$ values were determined by two-way ANOVA with Sidak's post hoc test. B ulk1/ulk2 DKO MEFs and ulk1/ulk2 DKO MEFs reconstituted with MYC-ULK1 were treated with different concentrations of Fin $56(0.2 \mu \mathrm{M}-10 \mu \mathrm{M})$ for 6 and $9 \mathrm{~h}$. After treatment, cell viability was measured using MTT assay. Results are shown as means + SD of two independent experiments performed in triplicates for each treatment. $P$ values were determined by two-way ANOVA with Sidak's post hoc test. C atg3 KO and Atg 3 WT MEFs were treated with different concentrations of Fin $56(0.2 \mu \mathrm{M}-10 \mu \mathrm{M})$ for 6 and $9 \mathrm{~h}$. After treatment, cell viability was measured using MTT assay. Results are shown as means + SD of two independent experiments performed in triplicates for each treatment. $P$ values were determined by two-way ANOVA with Sidak's post hoc test. ${ }^{*} p<0.05 ;{ }^{* *} p<0.01 ;{ }^{* * *} p<0.001 ;{ }^{* * * *} p$ $<0.0001$. In order to confirm knockdown or knockout, cells were lysed and cellular lysates were immunoblotted for ULK1, ATG3, and tubulin. The asterisk in $\mathbf{C}$ indicates an unspecific background band.

wanted to know if indeed macroautophagy is the mechanism mediating Fin56-induced GPX4 degradation. In a first approach, we again used the autophagy-incompetent atg3 KO MEFs. We observed that Fin56-induced GPX4 degradation is largely blocked in atg3 $\mathrm{KO}$ MEFs (Fig. 6C). In an alternative approach, we investigated the colocalization of GPX4 and LC3 by immunofluorescence. We found that treatment of FSH-GPX4-expressing MEFs with Fin56 induces the colocalization of GPX4 and LC3 (Figure S4).
As both ATG3 and LC3 are not required for CMA, we think that Fin56-induced GPX4 degradation depends on macroautophagy. Finally, we investigated if Fin56 also induces GPX4 degradation in the BC cell lines 253J and T24, and-if yes-if this can be blocked by autophagy inhibition. We observed that both were the case (Fig. 6D). Taken together, Fin56 induces GPX4 degradation in MEFs and BC cells, and inhibition of autophagy can block this degradation. 
A
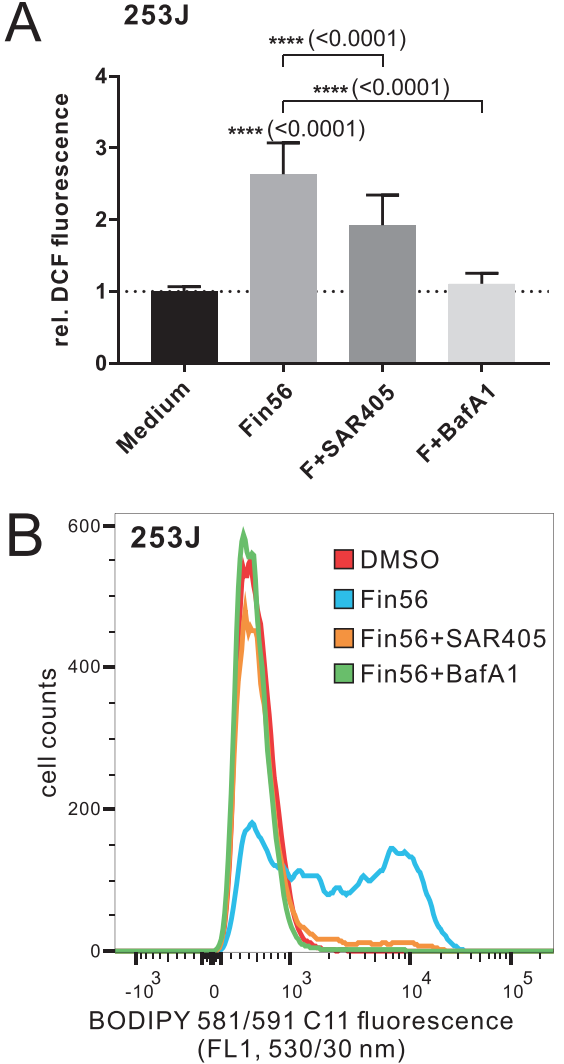

T24
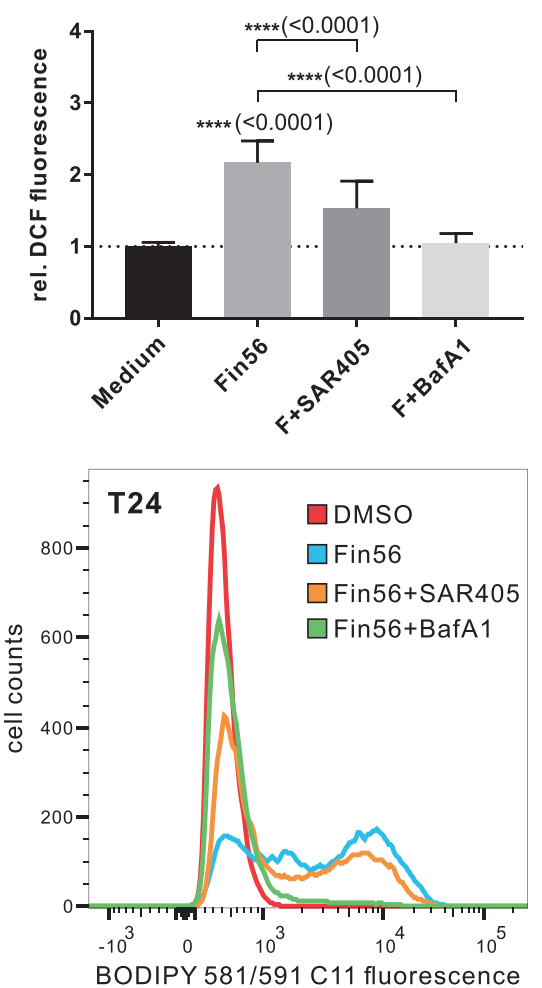

$(F L 1,530 / 30 \mathrm{~nm})$

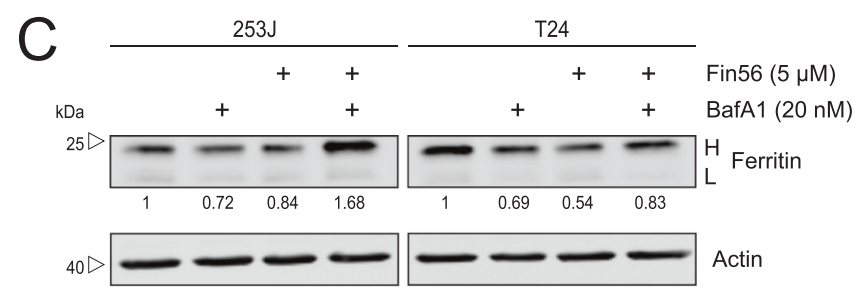

D 253J

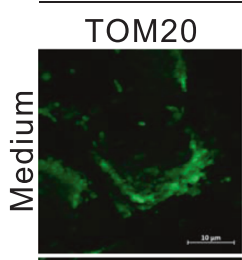

\section{LC3} Merged
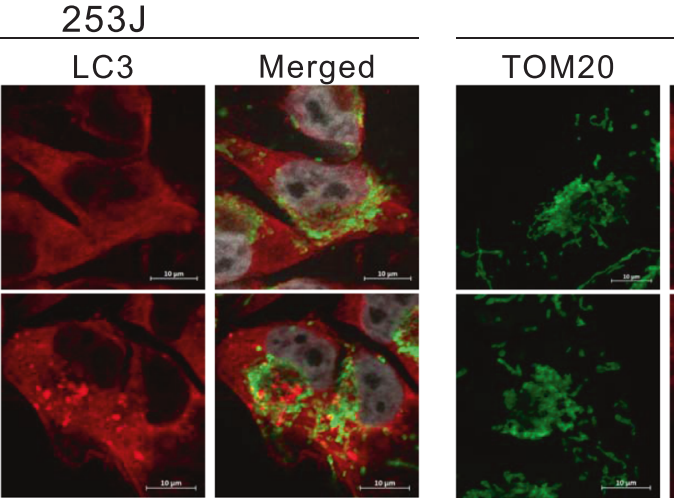

T24

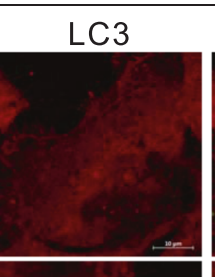

\section{Merged}
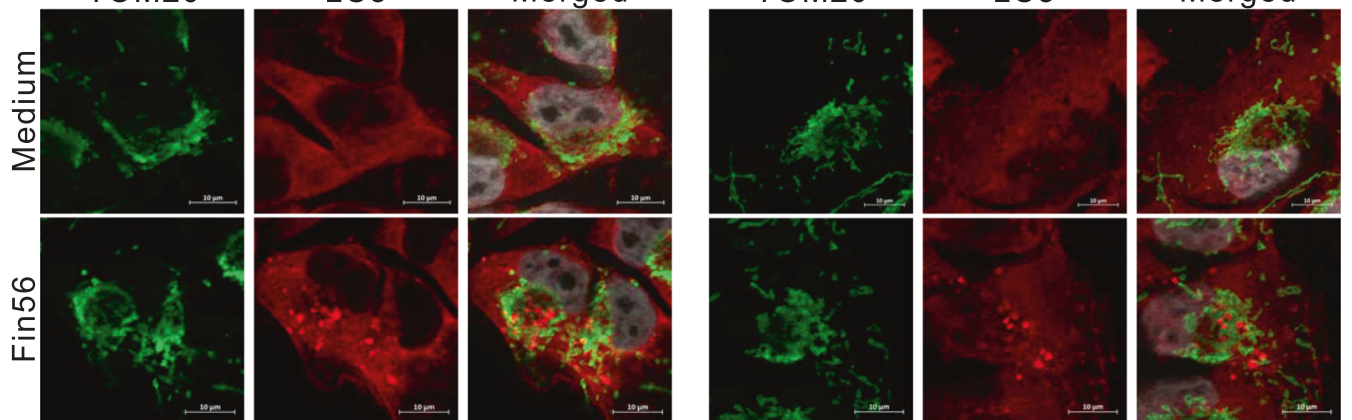

Fig. 5 Autophagy inhibition attenuates Fin56-induced oxidative stress. A $253 \mathrm{~J}$ and T24 cells were treated with Fin56 ( $2 \mu \mathrm{M})$, Fin56 + SAR405 $(2 \mu \mathrm{M})$ or Fin56 + BafA1 $(20 \mathrm{nM})$ for $4 \mathrm{~h}$. After treatment, cells were treated with $10 \mu \mathrm{M} \mathrm{H}_{2} \mathrm{DCFDA}$ for $30 \mathrm{~min}$. After that, DCF fluorescence was measured. Results are shown as means + SD of three independent experiments performed in triplicates for each treatment. $P$ values were determined by one-way ANOVA with Tukey's post hoc test. The respective $P$ values are depicted in the diagram. B $253 \mathrm{~J}$ and T24 cells were treated with DMSO, Fin56 $(2 \mu \mathrm{M})$, Fin56 + SAR405 $(2 \mu \mathrm{M})$ or Fin56 + BafA $1(20 \mathrm{nM})$ for $4 \mathrm{~h}$. Then, cells were incubated with $1.5 \mu \mathrm{M}$ BODIPY $581 / 591 \mathrm{C} 11$ for $30 \mathrm{~min}$ at $37^{\circ} \mathrm{C}$ before they were harvested by trypsinisation. Subsequently, cells were resuspended in $500 \mu \mathrm{L}$ PBS and analyzed by flow cytometry. Data were collected using the FL1 detector with a 530/30 BP filter. A total of 20,000 events was acquired for each sample. C $253 \mathrm{~J}$ and T24 cells were treated with Fin56 $(5 \mu \mathrm{M})$, BafA1 $(20 \mathrm{nM})$, or Fin56 + BafA 1 for $4 \mathrm{~h}$. After the incubation, cells were lysed and cellular lysates were immunoblotted for the indicated proteins $(H$, heavy chain; $L$, light chain). One representative immunoblot is shown. The quantifications are from two independent experiments. D 253J and T24 cells were grown on glass coverslips one day prior to treatment. The following day, cells were treated with $5 \mu \mathrm{M}$ Fin56 for $6 \mathrm{~h}$. After treatment, cells were fixed, permeabilized, and incubated with indicated antibodies. Nuclei were stained with DAPI. One representative image is shown. 
A

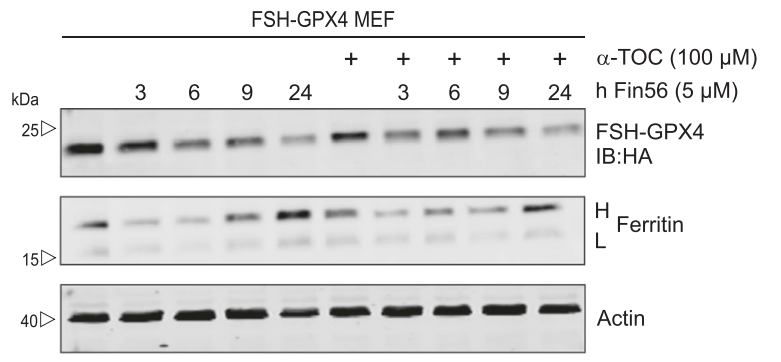

B

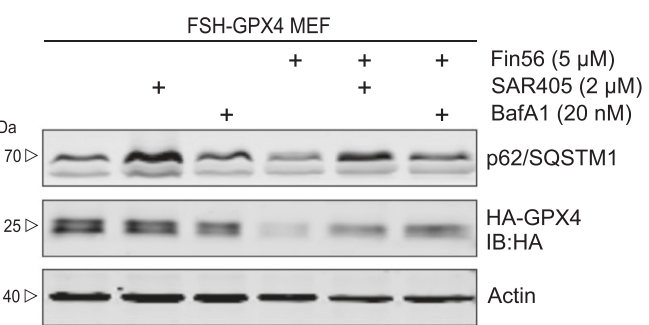

$\mathrm{C}$

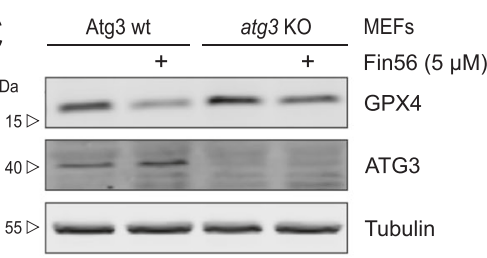

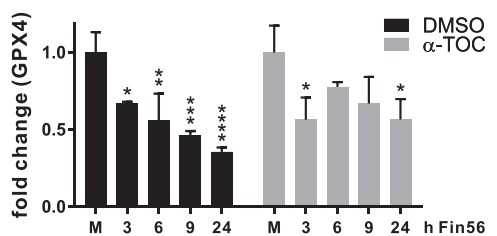
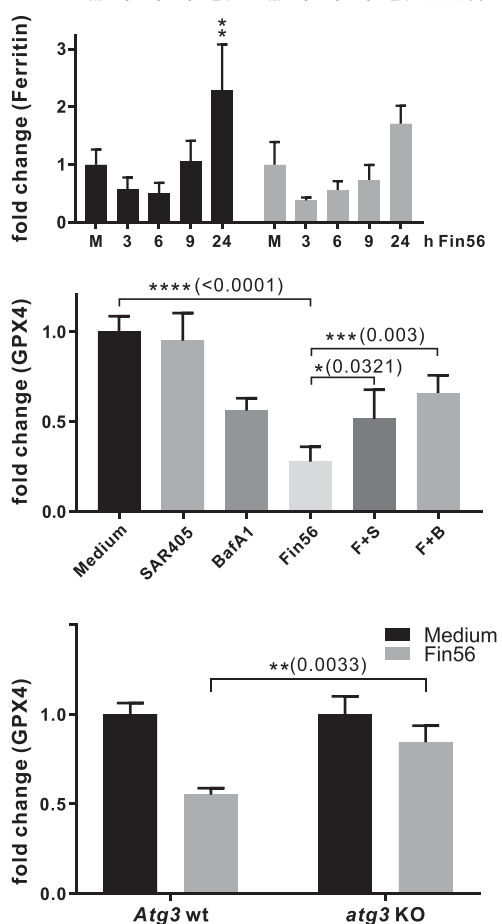
T24

$+\quad+\quad$ Fin56 $(5 \mu \mathrm{M})$

$+\quad+\quad$ BafA1 $(20 \mathrm{nM})$
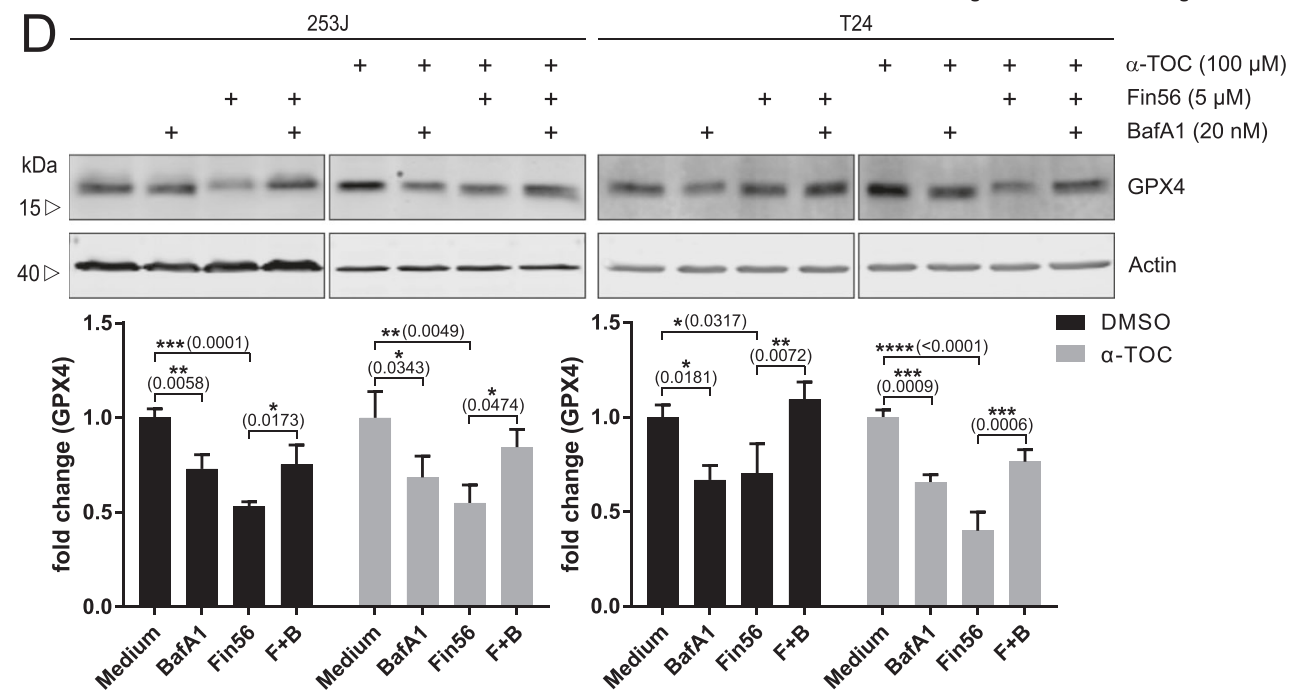

Fig. 6 Autophagy inhibition attenuates Fin56-induced GPX4 degradation. A FSH-GPX4 MEFs were left untreated (medium, M) or were treated with $5 \mu \mathrm{M}$ Fin 56 with or without $100 \mu \mathrm{M} \alpha$-tocopherol $(\alpha$-TOC) for $3,6,9$, or $24 \mathrm{~h}$. After the incubation, cells were lysed and cellular lysates were immunoblotted for the indicated proteins. One representative immunoblot is shown. The quantifications are from three independent experiments. $P$ values were determined by two-way ANOVA with Tukey's post hoc test (comparison always to medium-treated

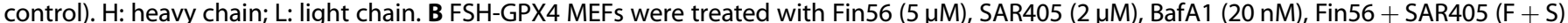
or Fin $56+$ BafA1 $(F+B)$ for $24 \mathrm{~h}$. After the incubation, cells were lysed and cellular lysates were immunoblotted for the indicated proteins. One representative immunoblot is shown. The quantifications are from five independent experiments. $P$ values were determined by one-way ANOVA with Tukey's post hoc test. C Atg3 wild-type or atg3 KO MEFs were treated with $5 \mu \mathrm{M}$ Fin56 for $24 \mathrm{~h}$. After the incubation, cells were lysed and cellular lysates were immunoblotted for the indicated proteins. One representative immunoblot is shown. The quantifications are from three independent experiments. $P$ values were determined by two-way ANOVA with Sidak's post hoc test. D 253J and T24 cells were treated with Fin $56(5 \mu \mathrm{M})$, BafA1 $(20 \mathrm{nM})$ or Fin56 + BafA1 $(\mathrm{F}+\mathrm{B})$ with or without $100 \mu \mathrm{M}$ of $\alpha-\mathrm{TOC}$ for $24 \mathrm{~h}$. After the incubation, cells were lysed and cellular lysates were immunoblotted for the indicated proteins. The quantifications are from three independent experiments. $P$ values were determined by one-way ANOVA with Tukey's post hoc test. ${ }^{*} p<0.05 ;{ }^{* *} p<0.01 ;{ }^{* * *} p<0.001 ;{ }^{* * * *} p<0.0001$. 
As we found that autophagy inhibition partially reduces Fin56induced cell death (Fig. 1) and GPX4 degradation (Fig. 6), we next wanted to know if these observations are correlated. First, we overexpressed human GPX4 in all four BC cell lines, and we detected a reduction of Fin56-induced cell death (Fig. 7A), confirming that the regulation of GPX4 levels is central to the control of ferroptosis in these cell lines. In RT-112 cells, which express high GPX4 levels endogenously (Fig. 7A and S1B), Fin56induced cell death was not completely preventable by GPX4 overexpression, supporting a previous report describing an additional Fin56-induced ferroptosis pathway independent of GPX4 degradation [14]. Next, we silenced GPX4 in all four BC cell lines (Fig. 7B). Notably, Fin56-induced reduction of cell viability was not sensitive to bafilomycin $A_{1}$ in $253 \mathrm{~J}$ and $T 24$ cells anymore, and silencing did not sensitize J82 and RT-112 cells. Apparently, the siRNA-mediated knockdown of GPX4 (transcriptional control) abolishes the autophagy-dependent part of Fin56-induced ferroptosis (control on the protein level). These data indicate that the sensitivity of $253 \mathrm{~J}$ and T224 cells to bafilomycin $A_{1}$ is-at least partially-caused by the Fin56-induced autophagic degradation of GPX4.

\section{mTOR inhibition synergistically sensitizes BCs to Fin56- induced ferroptosis}

As autophagy promotes Fin56-induced ferroptosis, we hypothesized that triggering both processes simultaneously might be a valuable strategy to increase the efficacy of killing BC cells. Here, we used mTOR inhibition to induce autophagy. We first validated that the mTOR inhibitor Torin 2 worked appropriately in our cellular systems. In FSH-GPX4-expressing MEFs, Torin 2 inhibited ULK1 Ser757 phosphorylation and decreased SQSTM1/p62 abundance, confirming the induction of autophagy (Fig. 8A). Torin 2 treatment also decreased GPX4 abundance (Fig. 8A), likely caused by the inhibition of protein synthesis. This finding motivated us to investigate the combined treatment of BC cells with Torin 2 and Fin56. To obtain $\mathrm{IC}_{50}$ values for combination analysis, we treated 253J and T24 cells with different concentrations of Torin 2 for $72 \mathrm{~h}$. Torin 2 displayed high cytotoxicity (Fig. 8B). Next, we analyzed GPX4 expression upon single and combined treatments with Torin 2 and Fin56. We observed that the single treatments reduced GPX4 protein levels, with the most-prominent effect for the combined treatment (Fig. $8 \mathrm{C}$ ). To investigate whether Torin 2 and Fin56 exhibit a synergistic effect on cytotoxicity, we performed isobologram analysis using the Chou-Talalay method (Fig. 8D) [49]. The combination of Torin 2 and Fin56 was synergistic at all tested concentrations in both BC cell lines (Fig. 8E and S5). Collectively, mTOR inhibition synergistically sensitizes BC cells to Fin56induced ferroptosis.

In conclusion, our results show that Fin56 induces autophagydependent ferroptosis in BC cells and that the pharmacological or genetic inhibition of autophagy protects cells from ferroptosis induced by Fin56, whereas the inhibition of mTOR synergistically increases Fin56-induced ferroptosis.

\section{DISCUSSION}

$\mathrm{BC}$ is one of the most-common genitourinary cancers. Even with improvements in multimodal treatment strategies, such as (neo) adjuvant chemo-/immunotherapy, the therapeutic effects are far from satisfactory. In this study, we found that the type 3 ferroptosis inducer Fin56 triggers autophagy-dependent ferroptosis in BC cells. We also found that autophagy inhibition attenuates ferritin and GPX4 degradation, and alleviates oxidative damage induced by Fin56. In addition, we showed that the combination of Fin56 and Torin 2 results in synergistic cytotoxicity in BC cells.

Accumulating evidence indicates that ferroptosis represents a type of autophagy-dependent cell death $[28,50]$. Several types of selective autophagy are involved in ferroptosis, such as clockophagy, ferritinophagy, lipophagy, and CMA [15, 29, 51, 52]. By evaluating the abundance of the autophagy markers LC3 and SQSTM1/p62 and the localization pattern of mRFP-EGFP-rLC3, we found that Fin56 induces autophagy in BC cells. Since LC3 and SQSTM1/p62 are common autophagy markers that participate in various types of autophagy, future studies will have to clarify whether Fin56 induces selective autophagy or just non-selective bulk autophagy.

Different types of ferroptosis inducers utilize different mechanisms to trigger this pathway. Fin56, a type 3 inducer, leads to ferroptosis mainly by promoting GPX4 degradation [14]. However, the mechanism(s) underlying this degradation are incompletely understood. Our study shows that Fin56 decreases GPX4 abundance, whereas autophagy inhibition at the nucleation stage (SAR405), at the expansion stage (ATG3 deficiency) or at the fusion stage (bafilomycin $A_{1}$ ) attenuates this degradation process. Similarly, we demonstrated that Fin56 treatment leads to ferritin degradation in both MEFs and human BC cells, and that the degradation was damped by autophagy inhibition. We also found that bafilomycin $A_{1}$ treatment alone reduces ferritin abundance (Fig. 5B). It has been reported that lysosomal vATPase inhibition causes functional iron deficiency by sequestering iron in the lysosomes and that iron deficiency causes ferritin degradation to maintain the balance of cellular iron [53]. As Fenton reaction is the main mechanism contributing to the generation of oxygen radicals, especially hydroxyl radicals, that in turn incite and propagate lipid peroxidation, careful control of iron levels is required to prevent Fenton reaction. GPX4 synthesis might decrease when cellular oxidative stress is relatively low. This could partially explain the reduction of GPX4 upon bafilomycin $A_{1}$ treatment alone (Fig. $6 \mathrm{~B}$ ).

The specific mechanisms of Fin56-induced GPX4 degradation have not been elucidated so far. Shimada et al. [14] reported that Fin56-induced GPX4 degradation could be prevented by the inhibition of acetyl-CoA carboxylase (ACC). For yeast, it has been recently shown that $A C C$-dependent lipogenesis promotes autophagy [54]. Accordingly, it might be that autophagy represents the missing link between ACC activity and GPX4 degradation. A recent report indicates that erastin promotes $C M A$-mediated degradation of GPX4 by increasing the levels of lysosome-associated membrane protein 2a [29]. Generally, this report is consistent with our results using Fin56, and highlights the important role of autophagy for ferroptosis. However, we also found changes in LC3 and SQSTM1/p62 levels, and these proteins are not necessarily involved in CMA. We also observed that GPX4 degradation was abolished in atg3 KO MEFs and that GPX4 colocalizes with LC3 upon Fin56 treatment, further suggesting that rather (macro)autophagy than CMA mediates Fin56-induced reduction of GPX4. Liu et al. [30] observed that RSL3 causes GPX4 degradation in human pancreatic cancer cells. Notably, hydroxychloroquine inhibited RSL3-induced GPX4 degradation only in PANC1 cells, but not in MIAPaCa2 cells [30]. Similarly, hydroxychloroquine could not prevent GPX4 degradation induced by mTOR inhibition [30]. These data indicate that GPX4 degradation does not rely on autophagy at least in the analyzed pancreatic cell lines. In our hands, Fin56-induced GPX4 degradation was inhibited by bafilomycin $A_{1}$, clearly supporting an autophagydependency for Fin56 in our model system. With regard to mTOR signaling, similar differences become obvious. It has been suggested that (i) mTORC1 inhibition synergizes with ferroptosis inducers to suppress tumor growth [31], (ii) mTORC1 inhibition decreases GPX4 levels $[30,31]$ and (iii) RSL3 blocks mTOR activation [30]. Whereas we also detect synergistic effects between Fin 56 and Torin 2 on the viability of BC cell lines and a Torin 2-mediated reduction of GPX4 levels, we do not observe an effect of Fin56 treatment on mTOR activation. It appears that Fin56 induces mTOR-independent autophagy, but that Fin56induced ferroptosis can be supported by mTOR inhibitionmediated autophagy. Collectively, these data already indicate a rather complex and possibly stimulus-/cell-dependent relationship 

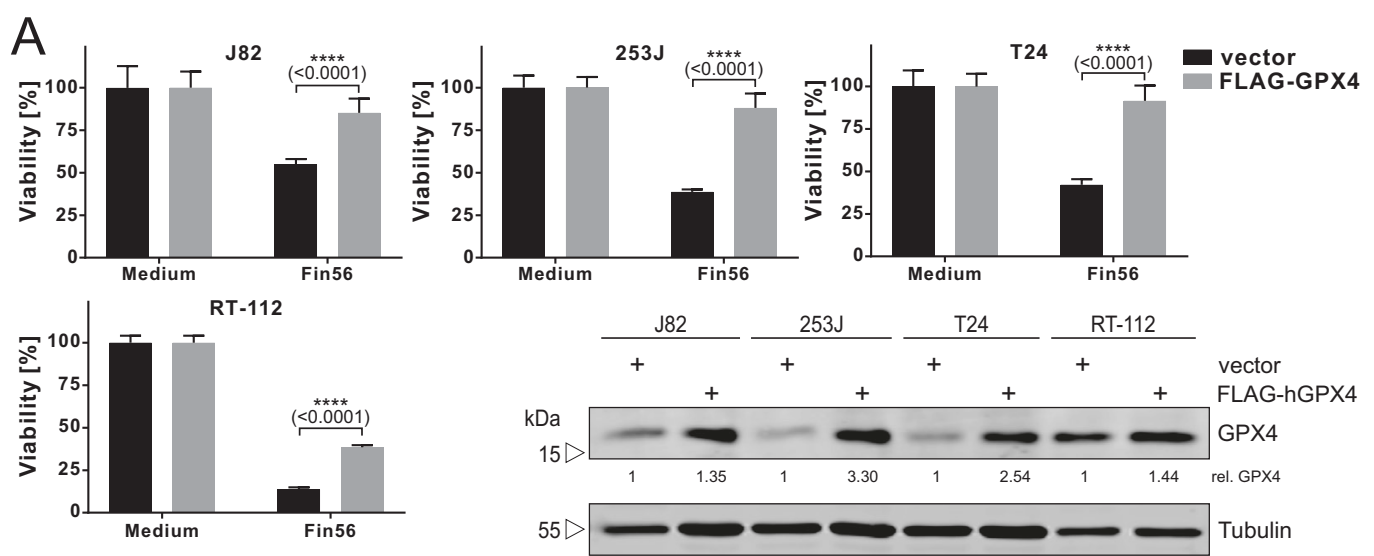

B J82
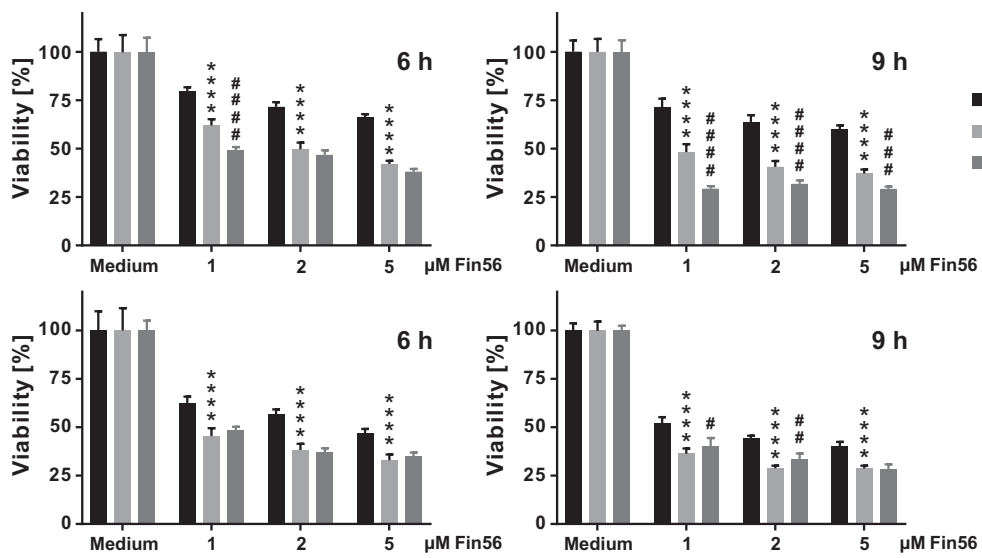

253J

T24
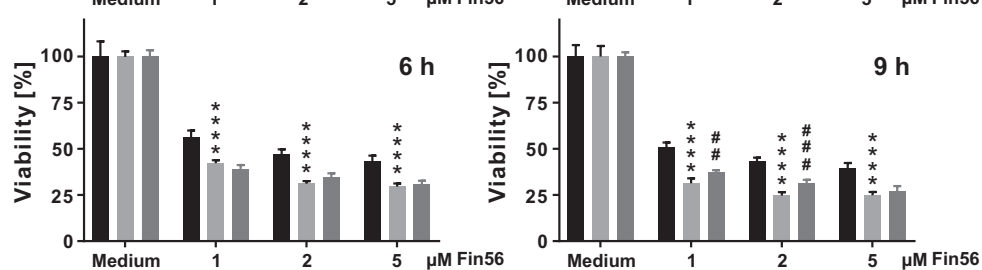

RT-112

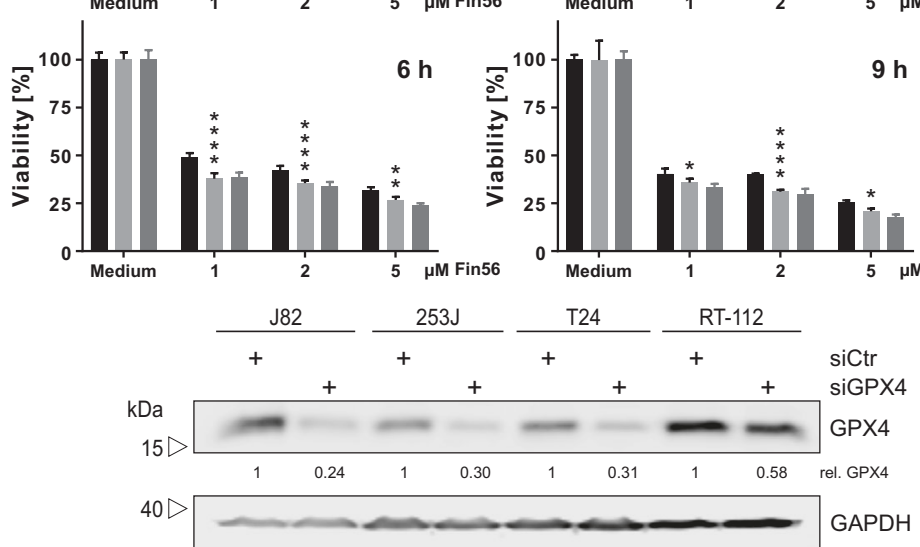

Fig. 7 GPX4 knockdown abolishes autophagy-dependency of Fin56-induced ferroptosis. A J82, 253J, T24, or RT-112 cells were transiently transfected with empty vector or cDNA encoding FLAG-hGPX4. After $30 \mathrm{~h}$, cells were seeded into 96-well plates for MTT cell viability assay and into six-well plates for immunoblotting. For cell viability assay, cells were treated with $5 \mu \mathrm{M}$ Fin 56 for $9 \mathrm{~h}$. Then, cell viability was measured using MTT assay. Results are shown as means + SD of two independent experiments performed in triplicates for each treatment. $P$ values were determined by two-way ANOVA with Sidak's post hoc test. Simultaneously, cells in six-well plates were lysed and cellular lysates were immunoblotted for GPX4 and tubulin. B J82, 253J, T24, or RT-112 cells were transfected with control or GPX4 siRNAs. After 48 h, cells were left

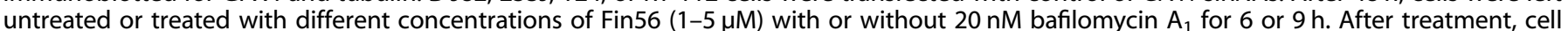
viability was measured using MTT assay. Results are shown as means + SD of two independent experiments performed in triplicates for each treatment. $P$ values were determined by one-way ANOVA with Tukey's post hoc test. The asterisk $\left(^{*}\right)$ represents the comparison between siCtr and siGPX4, whereas the octothorpe (\#) indicates the comparison between siGPX4 and siGPX4+BafA1. Successful knockdown was confirmed by immunoblotting for GPX4 and GAPDH. 
A
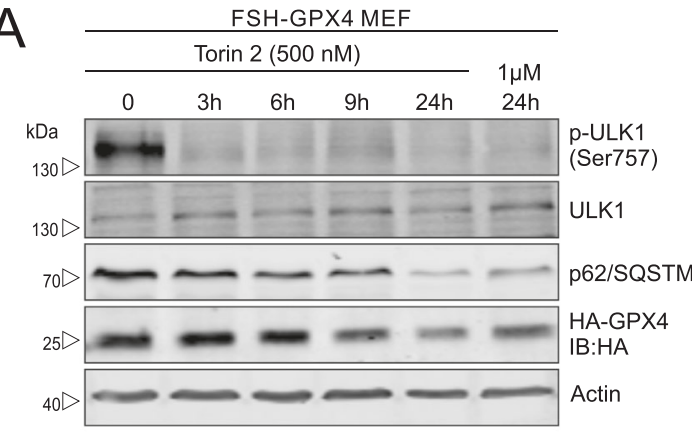

B

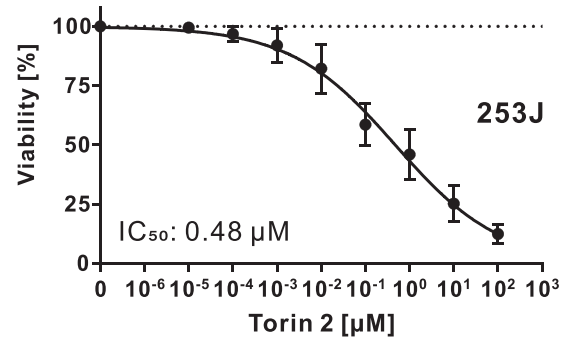

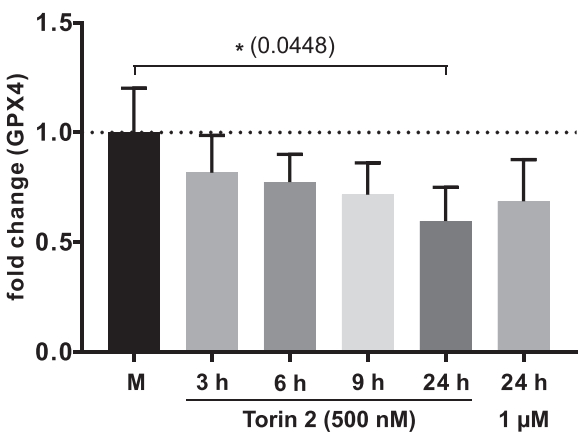

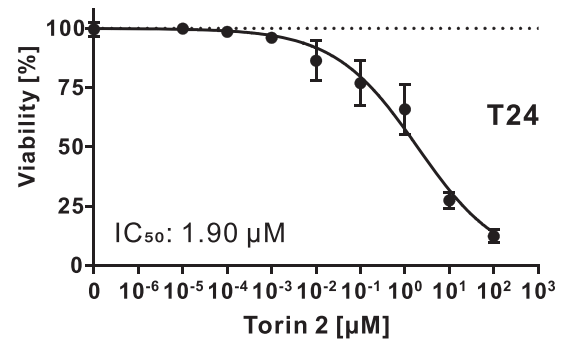

C
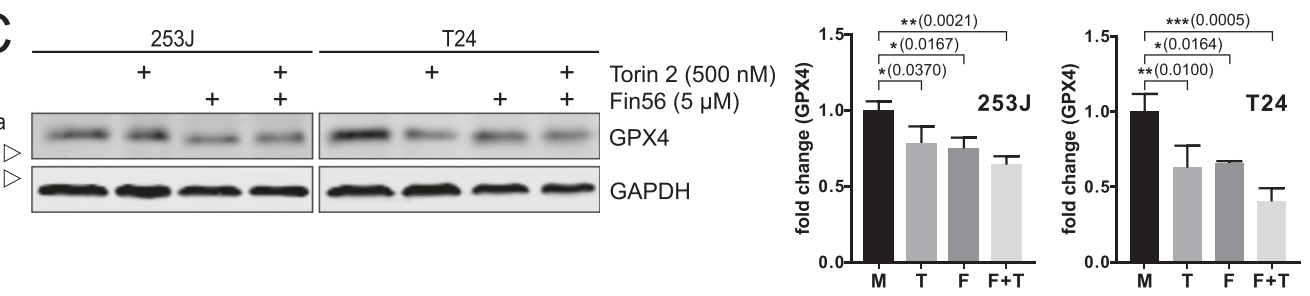

D
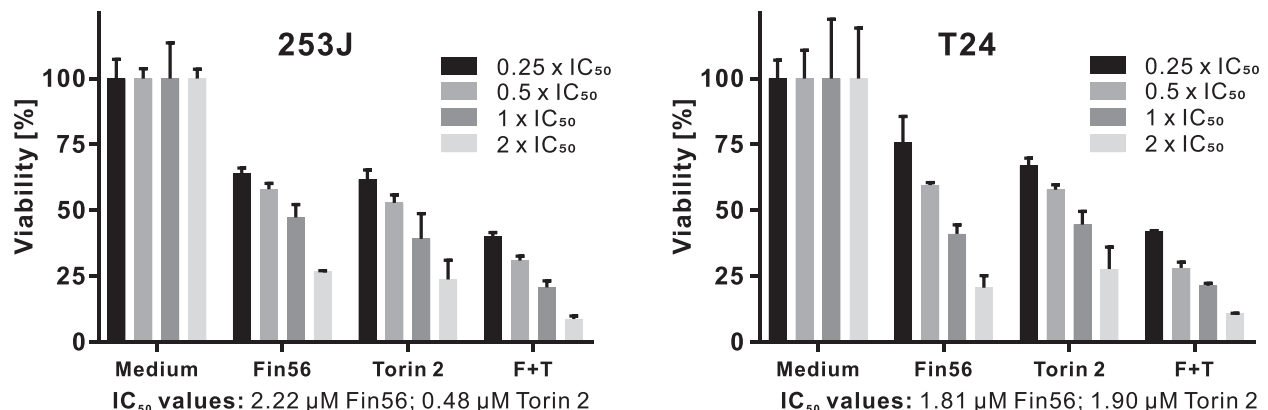

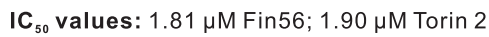
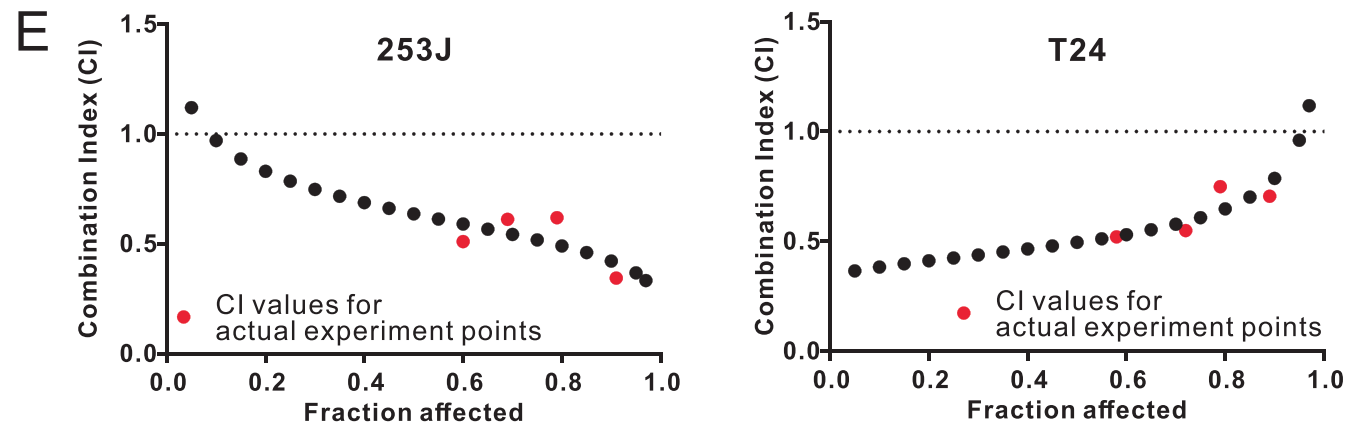

Fig. 8 mTOR inhibition synergistically sensitizes BCs to Fin56-induced ferroptosis. A FSH-GPX4 MEFs were left untreated (medium, M) or were treated with $500 \mathrm{nM}$ Torin 2 for 3, 6, 9, or $24 \mathrm{~h}$, or with $1 \mu \mathrm{M}$ Torin 2 for $24 \mathrm{~h}$. After the incubation, cells were lysed and cellular lysates were immunoblotted for the indicated proteins. One representative immunoblot is shown. The quantifications are from three independent experiments. $P$ values were determined by one-way ANOVA with Dunnett's post hoc test. B 253J and T24 cells were treated with different concentrations $(0.1 \mathrm{nM}-100 \mu \mathrm{M})$ of Torin 2 for $72 \mathrm{~h}$. After treatment, cell viability was measured using MTT assay. Results are shown as means \pm SD of three independent experiments performed in triplicates for each treatment. C 253J and T24 cells were left untreated (medium, M) or were treated with the indicated treatment $(500 \mathrm{nM}$ Torin $2, \mathrm{~T} ; 5 \mu \mathrm{M}$ Fin56, F) for $9 \mathrm{~h}$. After the incubation, cells were lysed and cellular lysates were immunoblotted for the indicated proteins. The quantifications are from three independent experiments. $P$ values were determined by one-way ANOVA with Tukey's post hoc test. D 253J and T24 cells were treated with $0.25 \times, 0.5 \times, 1 \times$, or $2 \times$ of the IC 50 values of the single substances for $72 \mathrm{~h}$. After treatment, cell viability was measured using MTT assay. Results are shown as means + SD of two independent experiments performed in sextuplicates for each treatment. E Combination indices (Cl) were calculated using the software CompuSyn. Synergism $(\mathrm{Cl}<1)$, additivism $(\mathrm{Cl}=1)$ and antagonism $(\mathrm{Cl}>1)$. 
between autophagy and ferroptosis. We observed autophagydependency in two of four tested BC cell lines, and this heterogeneity has also been reported for erastin and RSL3 with regard to LC3-II accumulation, SQSTM1/p62 downregulation, and GPX4 degradation [27]. Fin56-induced ferroptosis was inhibitable in two cell lines. Of note, erastin-induced cell death was bafilomycin $A_{1}$-sensitive in the identical two cell lines, indicating that the autophagy-dependency of ferroptosis signaling might be independent of the inducing stimulus. Generally, we observed different "categories" of Fin56-induced ferroptosis in our BC cells (Figure S6): (1) autophagy-dependent GPX4 degradation (253J, T24), (2) potentially autophagy-independent GPX4 degradation (J82; Figs. 1 and 7A), and (3) independent of GPX4 degradation (RT-112; Figs. 1 and 7A). It has already been proposed that Fin56induced ferroptosis can be mediated-next to GPX4 degradation - via squalene synthase activation and coenzyme $Q_{10}$ depletion [14], which might explain the observed phenotype in RT-112 cells. Future transcriptomic and proteomic analysis of our cell lines might reveal predictive factors for autophagy-dependent ferroptosis signaling.

Ferroptosis has attracted overwhelming interest in cancer research in recent years [55]. Since our results suggested that autophagy supports Fin56-induced ferroptosis, we validated the therapeutic benefit of the combination of Fin56 with autophagy activation, i.e., mTOR inhibition. Fin56 synergized with Torin 2 at all tested concentrations in BC cells. These findings constitute a promising proof-of-principle study to establish the combination of ferroptosis inducers with autophagy inhibitors for BC treatment.

\section{MATERIALS AND METHODS \\ Reagents}

Erastin (\#S7242), RSL3 (\#S8155), Fin56 (\#S8254), SAR405 (\#7682), Torin 2 (\#S2817) were purchased from Selleck Chemicals (Houston, TX, USA); bafilomycin $A_{1}$ (\#B1793), a-tocopherol (\#258024), and Liproxstatin-1 (\#SML1414) were purchased from Sigma-Aldrich (St. Louis, MO, USA). In addition, the following reagents were used: dimethyl sulfoxide (DMSO; AppliChem GmbH, \#A3672), milk powder (Carl Roth, \#T145.2), phosphate-buffered saline (PBS) (Thermo Fisher Scientific/Gibco, \#14190-094), 0.05\% trypsin/ EDTA solution (Thermo Fisher Scientific/Gibco, \#25300-062), puromycin (InvivoGen, \#ant-pr-1).

For immunoblotting, primary antibodies against SQSTM1/p62 (PROGEN Biotechnik, \#GP62-C), LC3 (Cell Signaling Technology, \#2775), ACTB/ $\beta$-actin (Sigma-Aldrich, \#A5316), phospho-mTOR Ser2448 (Cell Signaling Technology, \#2971), mTOR (Cell Signaling Technology, \#2972), phospho-ULK1 Ser757 (Cell Signaling Technology, \#6888), ULK1 (clone D8H5, Cell Signaling Technology, \#8054), tubulin (Sigma, \#T5168), ATG3 (Cell Signaling Technology, \#3415), SLC7A11 (previously described in ref. [56]), ferritin (Abcam, \#ab75973), HA Tag (clone 3F10, Roche Applied Science, \#11867423001), GPX4 (Abcam, \#ab125066), GAPDH (Abcam, \#ab8245) were used. IRDye 680- or IRDye 800-conjugated secondary antibodies (\#926-68077, \#926-68070/71, \#926-32210/11) were purchased from LI-COR Biosciences.

For immunofluorescence, primary antibodies against TOM20 (Santa Cruz Biotechnology, \#17764) and LC3B (MBL, \#PM036) were used. Alexa Fluor 488-conjugated and Alexa Fluor ${ }^{\circ}$ 647-conjugated secondary antibodies were purchased from Jackson ImmunoResearch Laboratories (\#115-545003 and \#111-605-003). DAPI was obtained from Roth (\#6335.1).

\section{Cell lines and cell culture}

All BC cell lines used in this research (kindly provided by Margaretha Skowron, Department of Urology, Heinrich Heine University Düsseldorf, Düsseldorf, Germany) were cultured in Dulbecco's Modified Eagle Medium (DMEM) (Thermo Fisher Scientific/Gibco, \#41965) supplemented with $10 \%$ fetal calf serum (FCS; Sigma-Aldrich, \#F0804) and $4.5 \mathrm{~g} / \mathrm{l}$ D-glucose at $37^{\circ} \mathrm{C}$ and $5 \% \mathrm{CO}_{2}$ humidified atmosphere. ulk1/ulk2 DKO MEFs (kindly provided by Tullia Lindsten, Memorial Sloan Kettering Cancer Center, New York City, USA), MYC-ULK1-expressing ulk1/ulk2 DKO MEFs (generation see below), atg3 KO and Atg3 WT MEFs (kindly provided by Masaaki Komatsu, Department of Organ and Cell Physiology, Juntendo University School of Medicine, Tokyo, Japan), and Flag-Strep-HA-GPX4 MEFs (previously described in ref. [47]) were cultured in DMEM supplemented with $10 \%$ FCS, $100 \mathrm{U} / \mathrm{ml}$ penicillin and $100 \mu \mathrm{g} / \mathrm{ml}$ streptomycin (Thermo Fisher Scientific/Gibco, 15140) at $37^{\circ} \mathrm{C}$ and $5 \% \mathrm{CO}_{2}$ humidified atmosphere.

\section{Cell viability assay}

Cell viability was measured using MTT assay. In all, 5000 cells per well were seeded on a 96-well plate one day prior to the experiment. The following day, the cells were treated with different compounds as described above. After that, $0.5 \mathrm{mg} / \mathrm{ml} \mathrm{MTT}$ (Roth, \#4022) was added to the cells, and cells were incubated at $37^{\circ} \mathrm{C}$ for $1 \mathrm{~h}$. Then the plates were centrifuged at $600 \mathrm{rcf}$ for $5 \mathrm{~min}$, and formazan crystals were dissolved in DMSO for $20 \mathrm{~min}$ in dark. The absorbance was measured at test $(570 \mathrm{~nm})$ and reference $(650 \mathrm{~nm})$ wavelengths using a microplate reader (BioTek, Synergy Mx). The mean of the absorbance of untreated control samples was set as $100 \%$.

\section{siRNA transfection}

For Ulk1 knockdown, wild-type MEFs (at 50-60\% confluence) in opti-MEM medium (Thermo Fisher Scientific, \#31985062) were transfected with DharmaFECT 1 reagent (Dharmacon, \#T-2001-03) using $25 \mathrm{nM}$ of ON-TARGETplus Mouse ULK1 siRNA-SMARTpool (Dharmacon, \#L-04015500-0010) or ON-TARGETplus Non-targeting Control Pool (Dharmacon, \#D-001810-10-20). For GPX4 knockdown, BCs at 70-80\% confluence were transfected with DharmaFECT 1 reagent using $25 \mathrm{nM}$ of ON-TARGETplus Human GPX4 siRNA-SMARTpool (Dharmacon, \#L-011676-00-0005) or nontargeting control pool. After $24 \mathrm{~h}$, cells were seeded for cell viability assay and immunoblotting.

\section{Retroviral transduction}

CDNA encoding human SLC7A11 was amplified from 253J CDNA by PCR using the following primers: fwd: CCCCCGTGTGTCCCTACTA, rev: GGCAGATTGCCAAGATCTCAA. Subsequently, the SLC7A11 CDNA harboring $5^{\prime}$ and $3^{\prime}$ overlap to pMSCVpuro sequences was amplified and directly cloned into pMSCVpuro (Clontech Laboratories, Takara Bio, \#631461) by sequence and ligation-independent cloning (SLIC; [57]). The following primers were used: HA-SLC7A11 fwd: GCCGCCACCATGTACCCATACGATGTTCCAGATTACGCTGTCAGAAAGCCTGTTGTG, HA-SLC7A11 rev: TACCCGGTAGAATTCTCATAACTT ATCTTCTTCTGG, pMSCV fwd: GAAGATAAGTTATGAGAATTCTACCGGGTAGG, and pMSCV rev: AGCGTAATCTGGAACATCGTATGGGTACATGGTGGCGGCGAATTCGTTAACCTCGAG. Human GPX4 and 3'-UTR of GPX4 were amplified from Jurkat cell CDNA using the following primers: fwd: GCTGGACGAGGGG AGGAG, rev: CACAAGGTAGCCAGGGGTG. They were subsequently cloned into pMSCVpuro. MYC-mULK1 cDNA was a gift from Do-Hyung Kim (Addgene plasmid \#31960; http://n2t.net/addgene:31960; RRID: Addgene_31960). To generate the pMSCVblast/MYC-mULK1 vector, MYCmULK1 was amplified with the primers $5^{\prime}$ gattaactcgagATGGAGCAAAAGCTC ATTTCTGAGG $3^{\prime}$ and $5^{\prime}$ ctagttgttaacTCAGGCATAGACACCACTCAGC $3^{\prime}$, digested with Xho1 and Hpa1 (Thermo Scientific, \#FD0694 and \#FD1034), and cloned into pMSCVblast digested with the same enzymes. Plat-E cells (kindly provided by Toshio Kitamura, Institute of Medical Science, University of Tokyo, Japan) were used as packaging cells and were transfected with the retroviral expression vectors pMSCVpuro/HA-SLC7A11, pMSCVpuro/mRFPEGFP-rLC3 (previously described in ref. [58]), pMSCVpuro/hGPX4 or pMSCVblast/MYC-mULK1 using FuGENE 6 (Roche, \#11988387001). For the retroviral infection of human cell lines, pVSVG was co-transfected into Plat-E cells. After $48 \mathrm{~h}$, human BC cells or ulk1/ulk2 DKO MEFs were incubated with the corresponding retroviral supernatants containing $3 \mu \mathrm{g} / \mathrm{ml}$ Polybrene (Sigma-Aldrich, \#H9268-106) and selected in a medium containing either $2.5 \mu \mathrm{g} / \mathrm{ml}$ puromycin (InvivoGen, \#ant-pr-1) or $35 \mu \mathrm{g} / \mathrm{ml}$ blasticidin (InvivoGen, \#ant-bl-05), respectively.

\section{Immunoblotting}

Cells were harvested by scraping and lysed in ice-cold lysis buffer $(20 \mathrm{mM}$ Tris/HCl, pH 7.5, $150 \mathrm{mM} \mathrm{NaCl}, 0.5 \mathrm{mM}$ EDTA, 1\% [v/v] Triton X-100, $1 \mathrm{mM}$ $\mathrm{Na}_{3} \mathrm{VO}_{4}, 10 \mu \mathrm{M} \mathrm{Na} \mathrm{MoO}_{4}, 2.5 \mathrm{mM} \mathrm{Na} \mathrm{P}_{2} \mathrm{O}_{7}, 10 \mathrm{mM} \mathrm{NaF}$ and protease inhibitor cocktail [Sigma-Aldrich, \#P2714]). Equal amounts of proteins were subjected to $8-15 \%$ sodium dodecyl sulphate-polyacrylamide gel electrophoresis. Proteins were then transferred to polyvinylidene fluoride membranes (Millipore, \#IPFL00010) and analyzed using the indicated primary antibodies and appropriate IRDye-conjugated secondary antibodies. Protein signals were detected using an Odyssey Infrared Imaging system (LI-COR Biosciences) and quantified using Image Studio lite 4.0 (LICOR Biosciences). 


\section{Immunofluorescence}

In all, $5 \times 10^{4}$ cells were plated on glass coverslips one day prior to treatment. After treatment, the cells were fixed on ice using $4 \%$ paraformaldehyde-FBS for $15 \mathrm{~min}$, quenched with $50 \mathrm{mM} \mathrm{NH} \mathrm{ml}_{4}$ for $15 \mathrm{~min}$, and permeabilized with $50 \mu \mathrm{g} / \mathrm{ml}$ digitonin (Roth, \#4005) for $5 \mathrm{~min}$. Subsequently, the cells stably expressing mRFP-EGFP-rLC3 were directly stained with DAPI. The other cells were blocked with $3 \%$ bovine serum albumin (Roth, \#8076)-PBS for $30 \mathrm{~min}$, and then incubated with indicated primary antibodies and corresponding secondary antibodies for $1 \mathrm{~h}$ each. Cells were embedded in ProLong Glass Antifade Mountant (Thermo Fisher Scientific, \#P36980) containing DAPI. For BODIPY 581/591 C11 staining, $2.4 \times 10^{4}$ of $253 \mathrm{~J}$ or T24 cells per well were seeded on ibidi $\mu$ Slide 8 Well (ibidi \#80826). The following day, cells were treated with Fin56 $(2 \mu \mathrm{M})$ or DMSO for $4 \mathrm{~h}$. After treatment, cells were incubated with $2 \mu \mathrm{M}$ BODIPY 581/591 C11 (Thermo Fisher Scientific, \#D3861) for $30 \mathrm{~min}$. Subsequently, cells were washed twice with PBS and subjected to microscopy. The Zeiss Axio Observer 7 fluorescence microscope (Zeiss, Köln, Germany) with a Plan Apochromat $\times 40 / 1.4$ oil-objective (Zeiss, Köln, Germany) was used for imaging. The images were quantified with ImageJ, and the macros used for quantification are listed in the supplementary methods.

\section{DCF assay}

In all, 5000 cells per well were seeded on a 96-well plate one day prior to the experiment. The following day, the cells were treated with different compounds as described above. After that, cells were washed once with PBS and treated with $10 \mu \mathrm{M} \mathrm{H_{2 } D C F D A}$ (Thermo Fisher Scientific, \#D399) at $37^{\circ} \mathrm{C}$ for $30 \mathrm{~min}$ in dark. Then, the cells were washed twice with PBS, and the DCF fluorescence was measured immediately using excitation $(490 \mathrm{~nm})$ and emission $(520 \mathrm{~nm})$ wavelengths using a microplate reader (BioTek, Synergy Mx).

\section{Assessment of lipid peroxidation using BODIPY 581/591 C11 by flow cytometry}

In all, $2 \times 10^{6}$ cells per well were seeded on 6-well dishes one day prior to the experiment. On the next day, cells were treated with the indicated concentration of Fin56 to induce ferroptosis. Cells were incubated with $1.5 \mu \mathrm{M}$ BODIPY $581 / 591 \mathrm{C} 11$ for $30 \mathrm{~min}$ at $37^{\circ} \mathrm{C}$ before they were harvested by trypsinisation. Subsequently, cells were resuspended in $500 \mu \mathrm{l}$ of fresh PBS and analyzed using an LSRFortessa flow cytometer (BD, Franklin Lakes, NJ, USA). The 488-nm laser was used for excitation. Data was collected using the FL1 detector with a 530/30 BP filter. A total of 20,000 events were acquired for each sample.

\section{Statistics}

For immunoblotting, the density of each protein band was divided by the average density of all bands of this protein. The ratios were normalized to the loading control, and fold changes were calculated by dividing each normalized ratio by the control line (fold change is 1 for the control lane, $n \geq 2$ ). The results are shown as mean \pm standard deviation in the bar diagrams and in the dose-response curves, and $P$ values were determined by ordinary one-way analysis of variance (ANOVA) with Dunnett's post hoc test. For cell viability assay, results are shown as mean \pm standard deviation in the bar diagrams, and $P$ values were determined by two-way ANOVA with Sidak's post hoc test. All IC $C_{50}$ values were calculated using GraphPad Prism 7.01. Compusyn 1.0 (ref. [59]) was used to perform isobologram analysis for the combination of two compounds. The resulting combination index $(\mathrm{Cl})$ values represent the combined effects of two compounds and are interpreted as synergistic $(\mathrm{Cl}<1)$, additive $(\mathrm{Cl}=1)$, or antagonistic $(\mathrm{Cl}>1)$ [59]. For the quantification of puncta in mRFP-EGFP-rLC3expressing cells, the images were quantified with ImageJ. The numbers of puncta in Fin56 treatment are normalized to the numbers of puncta in medium treatment. The results are shown as mean + standard deviation in the bar diagrams, and $P$ values were determined by two-way ANOVA with Sidak's post hoc test.

\section{DATA AVAILABILITY}

The data that support the findings of this study are available from the corresponding author, B.S., upon reasonable request.

\section{REFERENCES}

1. Bray F, Ferlay J, Soerjomataram I, Siegel RL, Torre LA, Jemal A. Global cancer statistics 2018: GLOBOCAN estimates of incidence and mortality worldwide for 36 cancers in 185 countries. CA Cancer J Clin. 2018;68:394-424.

2. Malone ER, Oliva M, Sabatini PJB, Stockley TL, Siu LL. Molecular profiling for precision cancer therapies. Genome Med. 2020;12:8.

3. Sjodahl G, Jackson CL, Bartlett JM, Siemens DR, Berman DM. Molecular profiling in muscle-invasive bladder cancer: more than the sum of its parts. J Pathol. 2019;247:563-73.

4. Ma KL, Liu J, Gao M, Wang CX, Ni J, Zhang Y, et al. Activation of mTOR contributes to foam cell formation in the radial arteries of patients with end-stage renal disease. Clin Nephrol. 2014;81:396-404.

5. Saxton RA, Sabatini DM. mTOR signaling in growth, metabolism, and disease. Cell 2017;168:960-76.

6. Murugan AK. mTOR: role in cancer, metastasis and drug resistance. Semin Cancer Biol. 2019;59:92-111.

7. Liu ST, Hui G, Mathis C, Chamie K, Pantuck AJ, Drakaki A. The current status and future role of the phosphoinositide 3 kinase/akt signaling pathway in urothelial cancer: an old pathway in the new immunotherapy era. Clin Genitourin Cancer. 2018;16:e269-e76.

8. Hua $\mathrm{H}$, Kong $\mathrm{Q}$, Zhang $\mathrm{H}$, Wang J, Luo $\mathrm{T}$, Jiang $\mathrm{Y}$. Targeting mTOR for cancer therapy. J Hematol Oncol. 2019;12:71.

9. Stockwell BR, Friedmann Angeli JP, Bayir H, Bush Al, Conrad M, Dixon SJ, et al. Ferroptosis: a regulated cell death nexus linking metabolism, redox biology, and disease. Cell 2017;171:273-85.

10. Liang $C$, Zhang $X$, Yang $M$, Dong $X$. Recent progress in ferroptosis inducers for cancer therapy. Adv Mater. 2019;31:e1904197.

11. Li D, Li Y. The interaction between ferroptosis and lipid metabolism in cancer. Signal Transduct Target Ther. 2020;5:108.

12. Li J, Cao F, Yin HL, Huang ZJ, Lin ZT, Mao N, et al. Ferroptosis: past, present and future. Cell Death Dis. 2020;11:88.

13. Jiang $X$, Stockwell BR, Conrad M. Ferroptosis: mechanisms, biology and role in disease. Nat Rev Mol Cell Biol. 2021;22:266-82.

14. Shimada K, Skouta R, Kaplan A, Yang WS, Hayano M, Dixon SJ, et al. Global survey of cell death mechanisms reveals metabolic regulation of ferroptosis. Nat Chem Biol. 2016;12:497-503.

15. Hou W, Xie Y, Song X, Sun X, Lotze MT, Zeh HJ 3rd, et al. Autophagy promotes ferroptosis by degradation of ferritin. Autophagy. 2016;12:1425-8.

16. Buccarelli M, Marconi M, Pacioni S, De Pascalis I, D'Alessandris QG, Martini M, et al. Inhibition of autophagy increases susceptibility of glioblastoma stem cells to temozolomide by igniting ferroptosis. Cell Death Dis. 2018;9:841.

17. Mizushima N, Komatsu M. Autophagy: renovation of cells and tissues. Cell 2011;147:728-41.

18. Parzych KR, Klionsky DJ. An overview of autophagy: morphology, mechanism, and regulation. Antioxid Redox Signal. 2014;20:460-73.

19. Yang Z, Klionsky DJ. Mammalian autophagy: core molecular machinery and signaling regulation. Curr Opin Cell Biol. 2010;22:124-31.

20. Alers S, Löffler AS, Wesselborg S, Stork B. Role of AMPK-mTOR-Ulk1/2 in the regulation of autophagy: cross talk, shortcuts, and feedbacks. Mol Cell Biol. 2012;32:2-11.

21. Stork B, Dengjel J. Study of ULK1 catalytic activity and its regulation. Methods Enzymol. 2017;587:391-404.

22. Levy JMM, Towers CG, Thorburn A. Targeting autophagy in cancer. Nat Rev Cancer. 2017;17:528-42.

23. Amaravadi RK, Kimmelman AC, Debnath J. Targeting autophagy in cancer: recent advances and future directions. Cancer Discov. 2019;9:1167-81.

24. Bialik S, Dasari SK \& Kimchi A. Autophagy-dependent cell death - where, how and why a cell eats itself to death. J Cell Sci. 2018;131:jcs215152.

25. Denton D, Kumar S. Autophagy-dependent cell death. Cell Death Differ 2019;26:605-16.

26. Chen X, Yu C, Kang R, Kroemer G, Tang D. Cellular degradation systems in ferroptosis. Cell Death Differ. 2021;28:1135-48.

27. Li J, Liu J, Xu Y, Wu R, Chen X, Song X, et al. Tumor heterogeneity in autophagydependent ferroptosis. Autophagy. 2021;1-14.

28. Liu J, Kuang F, Kroemer G, Klionsky DJ, Kang R, Tang D. Autophagy-dependent ferroptosis: machinery and regulation. Cell Chem Biol. 2020;27:420-35.

29. Wu Z, Geng Y, Lu X, Shi Y, Wu G, Zhang M, et al. Chaperone-mediated autophagy is involved in the execution of ferroptosis. Proc Natl Acad Sci USA. 2019;116:2996-3005.

30. Liu Y, Wang Y, Liu J, Kang R, Tang D. Interplay between MTOR and GPX4 signaling modulates autophagy-dependent ferroptotic cancer cell death. Cancer gene Ther. 2021;28:55-63.

31. Zhang Y, Swanda RV, Nie L, Liu X, Wang C, Lee H, et al. mTORC1 couples cyst(e) ine availability with GPX4 protein synthesis and ferroptosis regulation. Nat Commun. 2021;12:1589. 
32. Lei $P$, Bai T, Sun Y. Mechanisms of ferroptosis and relations with regulated cell death: a review. Front Physiol. 2019;10:139.

33. Xie $Y$, Hou W, Song $X, Y u$ Y, Huang J, Sun $X$, et al. Ferroptosis: process and function. Cell Death Differ. 2016;23:369-79.

34. Yu H, Guo P, Xie X, Wang Y, Chen G. Ferroptosis, a new form of cell death, and its relationships with tumourous diseases. J Cell Mol Med. 2017;21:648-57.

35. Conrad M, Sato H. The oxidative stress-inducible cystine/glutamate antiporter, system x (c) (-): cystine supplier and beyond. Amino Acids. 2012;42:231-46.

36. Lewerenz J, Hewett SJ, Huang Y, Lambros M, Gout PW, Kalivas PW, et al. The cystine/ glutamate antiporter system $\mathrm{x}(\mathrm{c})(-)$ in health and disease: from molecular mechanisms to novel therapeutic opportunities. Antioxid Redox Signal. 2013;18:522-55.

37. Mizushima N, Yoshimori T, Levine B. Methods in mammalian autophagy research. Cell 2010;140:313-26.

38. He C, Klionsky DJ. Regulation mechanisms and signaling pathways of autophagy. Annu Rev Genet. 2009;43:67-93.

39. Hieke N, Löffler AS, Kaizuka T, Berleth N, Böhler P, Driessen S, et al. Expression of a ULK1/2 binding-deficient ATG13 variant can partially restore autophagic activity in ATG13-deficient cells. Autophagy 2015;11:1471-83.

40. Kim YC, Guan KL. mTOR: a pharmacologic target for autophagy regulation. J Clin Invest. 2015;125:25-32.

41. Chiang GG, Abraham RT. Phosphorylation of mammalian target of rapamycin (mTOR) at Ser-2448 is mediated by p70S6 kinase. J Biol Chem. 2005;280:25485-90.

42. Kim J, Kundu M, Viollet B, Guan KL. AMPK and mTOR regulate autophagy through direct phosphorylation of Ulk1. Nat Cell Biol. 2011;13:132-41.

43. Sou YS, Waguri S, Iwata J, Ueno T, Fujimura T, Hara T, et al. The Atg8 conjugation system is indispensable for proper development of autophagic isolation membranes in mice. Mol Biol Cell. 2008;19:4762-75.

44. Karlsson M, Kurz T, Brunk UT, Nilsson SE, Frennesson $\mathrm{Cl}$. What does the commonly used DCF test for oxidative stress really show? Biochem J. 2010;428:183-90.

45. Arosio P, Elia L, Poli M. Ferritin, cellular iron storage and regulation. IUBMB Life. 2017:69:414-22.

46. Ashrafi G, Schwarz TL. The pathways of mitophagy for quality control and clearance of mitochondria. Cell Death Differ. 2013;20:31-42.

47. Ingold I, Berndt C, Schmitt S, Doll S, Poschmann G, Buday K, et al. Selenium utilization by GPX4 is required to prevent hydroperoxide-induced ferroptosis. Cell 2018;172:409-22. e21

48. Mannes AM, Seiler A, Bosello V, Maiorino M, Conrad M. Cysteine mutant of mammalian GPx4 rescues cell death induced by disruption of the wild-type selenoenzyme. FASEB J. 2011;25:2135-44.

49. Roell KR, Reif DM, Motsinger-Reif AA. An introduction to terminology and methodology of chemical synergy-perspectives from across disciplines. Front Pharm. 2017;8:158.

50. Zhou B, Liu J, Kang R, Klionsky DJ, Kroemer G, Tang D. Ferroptosis is a type of autophagy-dependent cell death. Semin Cancer Biol. 2020;66:89-100.

51. Yang $M$, Chen P, Liu J, Zhu S, Kroemer G, Klionsky DJ, et al. Clockophagy is a novel selective autophagy process favoring ferroptosis. Sci Adv. 2019;5:eaaw2238.

52. Bai Y, Meng L, Han L, Jia Y, Zhao Y, Gao H, et al. Lipid storage and lipophagy regulates ferroptosis. Biochem Biophys Res Commun. 2019;508:997-1003.

53. Yambire KF, Rostosky C, Watanabe T, Pacheu-Grau D, Torres-Odio S, SanchezGuerrero A, et al. Impaired lysosomal acidification triggers iron deficiency and inflammation in vivo. Elife. 2019;8:e51031.

54. Gross AS, Zimmermann A, Pendl T, Schroeder S, Schoenlechner H, Knittelfelder O, et al. Acetyl-CoA carboxylase 1-dependent lipogenesis promotes autophagy downstream of AMPK. J Biol Chem. 2019;294:12020-39.

55. Friedmann Angeli JP, Krysko DV, Conrad M. Ferroptosis at the crossroads of canceracquired drug resistance and immune evasion. Nat Rev Cancer. 2019;19:405-14.

56. Doll S, Freitas FP, Shah R, Aldrovandi M, da Silva MC, Ingold I, et al. FSP1 is a glutathione-independent ferroptosis suppressor. Nature 2019;575:693-8.

57. Li MZ, Elledge SJ. Harnessing homologous recombination in vitro to generate recombinant DNA via SLIC. Nat Methods. 2007;4:251-6.

58. Alers S, Löffler AS, Paasch F, Dieterle AM, Keppeler H, Lauber K, et al. Atg13 and FIP200 act independently of Ulk1 and Ulk2 in autophagy induction. Autophagy 2011;7:1423-33.

59. Chou TC. Drug combination studies and their synergy quantification using the Chou-Talalay method. Cancer Res. 2010;70:440-6.

\section{ACKNOWLEDGEMENTS}

We thank Do-Hyung Kim (Department of Biochemistry, Molecular Biology and Biophysics, University of Minnesota, Minneapolis, USA) for providing MYC-mULK1 cDNA (Addgene plasmid \#31960; http://n2t.net/addgene:31960; RRID:
Addgene_31960). We thank Margaretha Skowron (Department of Urology, Medical Faculty, Heinrich Heine University, Düsseldorf, Germany) for providing J82, 253J, T24, and RT-112 bladder carcinoma cells. We thank Tullia Lindsten (Memorial Sloan Kettering Cancer Center, New York City, USA) for providing wild-type and ulk1/ulk2 DKO mouse embryonic fibroblasts. We thank Masaaki Komatsu (Department of Organ and Cell Physiology, Juntendo University School of Medicine, Tokyo, Japan) for providing wild-type and atg3 knockout mouse embryonic fibroblasts. We thank Toshio Kitamura (Institute of Medical Science, University of Tokyo, Japan) for providing Plat-E cells.

\section{AUTHOR CONTRIBUTIONS}

Y.S. designed the experiments and generated cell lines expressing SLC7A11 or mRFPEGFP-rLC3. Y.S. performed cell viability assay, DCF assay, immunofluorescence, and immunoblot analyses. M.J.M. generated MYC-ULK1 expressing ulk1/ulk2 DKO MEFs. W.W., D.S., J.D., F.S., L.B., A.F., S.A., N.B., M.J.M., M.C., and C.B. gave technical support. Y.S., N.B., S.W., M.C., C.B., and B.S. analyzed and interpreted the data. Y.S. and B.S. wrote the manuscript. B.S. supervised the project. All authors discussed the results and commented on the manuscript. B.S. and S.W. are supported by the Deutsche Forschungsgemeinschaft (DFG) (GRK 2158, GRK 2578), and by the Düsseldorf School of Oncology (funded by the Comprehensive Cancer Center Düsseldorf/Deutsche Krebshilfe and the Medical Faculty of the Heinrich Heine University Düsseldorf). B.S. is additionally supported by the DFG (STO 864/4-3, STO 864/5-1, STO 864/6-1). C.B. is supported by the DFG (BE 3259/5-2, GRK 2578). M.C. is supported by the DFG (CO 291/7-1, CO 291/9-1, CO 291/10-1), the German Federal Ministry of Education and Research (BMBF) VIP+ program NEUROPROTEKT (03VP04260), the Ministry of Science and Higher Education of the Russian Federation (075-15-2019-1933) and the Else Kröner-Fresenius-Stiftung and has further received funding from the European Research Council (ERC) under the European Union's Horizon 2020 research and innovation program (grant agreement no. GA 884754).

\section{FUNDING}

Open Access funding enabled and organized by Projekt DEAL.

\section{COMPETING INTERESTS}

M.C. holds patents for the liproxstatin class of compounds and is co-founder and shareholder of ROSCUE Therapeutics $\mathrm{GmbH}$.

\section{ADDITIONAL INFORMATION}

Supplementary information The online version contains supplementary material available at https://doi.org/10.1038/s41419-021-04306-2.

Correspondence and requests for materials should be addressed to Björn Stork.

Reprints and permission information is available at http://www.nature.com/ reprints

Publisher's note Springer Nature remains neutral with regard to jurisdictional claims in published maps and institutional affiliations.

Open Access This article is licensed under a Creative Commons Attribution 4.0 International License, which permits use, sharing, adaptation, distribution and reproduction in any medium or format, as long as you give appropriate credit to the original author(s) and the source, provide a link to the Creative Commons license, and indicate if changes were made. The images or other third party material in this article are included in the article's Creative Commons license, unless indicated otherwise in a credit line to the material. If material is not included in the article's Creative Commons license and your intended use is not permitted by statutory regulation or exceeds the permitted use, you will need to obtain permission directly from the copyright holder. To view a copy of this license, visit http://creativecommons. org/licenses/by/4.0/.

(c) The Author(s) 2021 